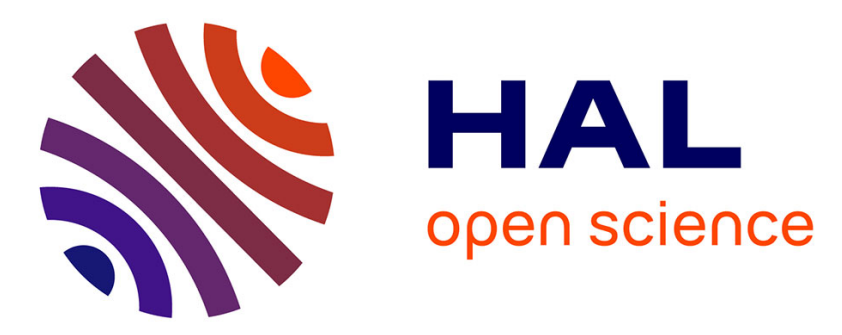

\title{
Global stability and control of the confined turbulent flow past a thick flat plate
}

\author{
Marco Carini, Christophe Airiau, A. Debien, O. Léon, J.O. Pralits
}

\section{To cite this version:}

Marco Carini, Christophe Airiau, A. Debien, O. Léon, J.O. Pralits. Global stability and control of the confined turbulent flow past a thick flat plate. Physics of Fluids, 2017, 29 (024102), 19 p. 10.1063/1.4974069 . hal-01474193

\section{HAL Id: hal-01474193 \\ https://hal.science/hal-01474193}

Submitted on 28 Sep 2017

HAL is a multi-disciplinary open access archive for the deposit and dissemination of scientific research documents, whether they are published or not. The documents may come from teaching and research institutions in France or abroad, or from public or private research centers.
L'archive ouverte pluridisciplinaire HAL, est destinée au dépôt et à la diffusion de documents scientifiques de niveau recherche, publiés ou non, émanant des établissements d'enseignement et de recherche français ou étrangers, des laboratoires publics ou privés. 


\section{Open Archive TOULOUSE Archive Ouverte (OATAO)}

OATAO is an open access repository that collects the work of Toulouse researchers and makes it freely available over the web where possible.

This is a publisher's version published in : http://oatao.univ-toulouse.fr/ Eprints ID : 18313

To link to this article : DOI: $10.1063 / 1.4974069$

URL : http://dx.doi.org/10.1063/1.4974069

To cite this version : Carini, Marco and Airiau, Christophe and Debien, Antoine and Léon, Olivier and Pralits, J.O. Global stability and control of the confined turbulent flow past a thick flat plate. (2017) Physics of Fluids, vol. 29 (n 2). pp. 024102/1-024102/19. ISSN 1070-6631

Any correspondence concerning this service should be sent to the repository administrator: staff-oatao@ listes-diff.inp-toulouse.fr 


\title{
Global stability and control of the confined turbulent flow past a thick flat plate
}

\author{
M. Carini, ${ }^{1}$ C. Airiau, ${ }^{1}$ A. Debien, ${ }^{2}$ O. Léon, ${ }^{2}$ and J. O. Pralits ${ }^{3, a)}$ \\ ${ }^{1}$ Université de Toulouse, Institut de Mécaniques des Fluides de Toulouse, UMR 5502 CNRS/INPT-UPS, \\ Allée du Professeur Camille Soula, F-31400 Toulouse, France \\ ${ }^{2}$ Aerodynamics and Energetics Modelling Department, ONERA, 2 Avenue Edouard Belin, \\ 31000 Toulouse, France \\ ${ }^{3}$ Dipartimento di Ingegneria Civile, Chimica e Ambientale, Università degli Studi di Genova, \\ via Montallegro 1, 16145 Genova, Italy
}

(Received 20 September 2016; accepted 31 December 2016; published online 9 February 2017)

\begin{abstract}
This article investigates the structural stability and sensitivity properties of the confined turbulent wake behind an elongated D-shaped cylinder of aspect-ratio 10 at $R e=32000$. The stability analysis is performed by linearising the incompressible Navier-Stokes equations around the numerically computed and the experimentally measured mean flows. We found that the vortex-shedding frequency is very well captured by the leading unstable global mode, especially when the additional turbulent diffusion is modelled in the stability equations by means of a frozen eddy-viscosity approach. The sensitivity maps derived from the computed and the measured mean flows are then compared, showing a good qualitative agreement. The careful inspection of their spatial structure highlights that the highest sensitivity is attained not only across the recirculation bubble but also at the body blunt-edge, where tiny pockets of maximum receptivity are found. The impact of the turbulent diffusion on the obtained results is investigated. Finally, we show how the knowledge of the unstable adjoint global mode of the linearised mean-flow dynamics can be exploited to design an active feedback control of the unsteady turbulent wake, which leads, under the adopted numerical framework, to completely suppress its low-frequency oscillation. Published by AIP Publishing. [http://dx.doi.org/10.1063/1.4974069]
\end{abstract}

\section{INTRODUCTION}

Understanding and controlling the flow in the wake of geometries which give rise to large scale unstable structures, such as for instance vortex shedding, is of paramount importance in many engineering applications. This is the case, for example, of the separated flow over a wing, resulting in unwanted aerodynamic loading, and of the unstable wake behind a bluff body, where the flow unsteadiness increases the aerodynamic drag and becomes a source of acoustic noise. The idea to use passive or active control strategies to improve the performance as well as the efficiency of certain applications is far from new and has been extensively investigated both numerically and experimentally. ${ }^{1-3}$

Numerical modelling of fluid flows and mathematically rigorous theories for their control ${ }^{4,5}$ have been usually first tested and verified in simplified conditions, at low or moderate Reynolds numbers. ${ }^{6-9}$ Within this framework, the concept of structural sensitivity has gained interest with applications to a large variety of globally unstable flows. ${ }^{10-15}$ Indeed this linearised approach allows one to predict, beforehand, the effective positioning of a flow disturbance, i.e., a passive device, able to shift the vortex-shedding frequency or even to completely suppress the global instability of the flow. A remarkable example is represented by the possibility to qualitatively recover the well-known experimental control map of

\footnotetext{
a) Author to whom correspondence should be addressed. Electronic mail: jan.pralits@unige.it
}

Strykowski and Sreenivasan ${ }^{16}$ for the circular cylinder wake, as shown by Marquet, Sipp and Jacquin. ${ }^{17}$ At the same time, a main concern is that the underlying theory is limited to lowReynolds number flows near the instability threshold, which often makes these techniques of little interest from practical and industrial viewpoint.

Despite the lack of a rigorous mathematical foundation, over the last few years, the above approach has been applied to turbulent bluff-body wakes, heuristically based on the linear stability analysis of time-averaged mean flows. For various flow configurations, past studies ${ }^{18-20}$ have shown that either linearised Euler or Navier-Stokes equations around the mean flow successfully exhibit a mildly unstable global mode with approximately the same frequency of the unsteady flow. These results are reminiscent of the seminal work of Malkus, ${ }^{21}$ first conjecturing the marginal stability of the mean-flow field, a conjecture which has been pursued by Mantič-Lugo, Arratia and Gallaire ${ }^{22}$ to build-up an effective self-consistent model of the nonlinear saturation mechanisms of the circular cylinder wake up to $R e=110$. The marginal stability criterion becomes indeed exact in the case of pure monochromatic oscillations of the flow, as shown by Turton, Tuckerman and Berkley, ${ }^{23}$ and further theoretical insight to the more general case of a broadband spectrum has been recently given by Beneddine $e$ t al. ${ }^{24}$ based on the singular-value analysis of the resolvent operator associated with the mean-flow linearised equations, with focus to high Reynolds number weakly non-parallel flows.

A first attempt at pushing forward the structural sensitivity analysis in the context of turbulent flows has been made by 
Meliga, Pujals and Serre. ${ }^{25}$ In their study, the authors computed the time-averaged solution of the unsteady two-dimensional Reynolds-Averaged Navier-Stokes (RANS) equations for the flow past a D-shaped cylinder at $R e=13000$ and then performed a sensitivity analysis of the obtained mean flow by linearising both the flow and the turbulence model equations. In analogy with the studies of the circular cylinder wake at low Reynolds numbers, the frequency control map computed by the authors for the considered turbulent flow was in close agreement with the one experimentally obtained by Parezanović and $\mathrm{Cadot}^{26}$ through extensive measurements performed for different positions of the secondary cylinder. Later, similar results were reproduced by Mettot, Sipp and Bézard ${ }^{27}$ by means of a simpler approach, where the linearisation is restricted to the flow equations only, thus making the whole procedure independent from the turbulence modelling. In the experimental study by Camarri, Fallenius and Fransson ${ }^{28}$ the same approach is applied to the mean wake behind a circular cylinder with transpiration at $R e=3500$. Although the stability and sensitivity analyses of experimental data pose some computational difficulties, the authors have shown that this approach can predict, with good accuracy, not only the vortexshedding frequency but also its variation with respect to the transpiration parameter, based on the induced modifications of the mean flow.

The present study further explores the possibility to capture and control low-frequency unsteadiness in turbulent bluffbody wakes based on the stability properties of the inherent mean flow. To this purpose, we consider the confined incompressible flow past an elongated D-shaped cylinder at $R e=32000$ by means of both numerical and experimental investigations. In analogy with the aforementioned studies, ${ }^{25,27}$ numerical simulations are undertaken in the RANS framework while the experimental measurements are performed by means of Particle Image Velocimetry (PIV) in the near-wake region of the flow. Notwithstanding that the accurate and reliable numerical prediction of turbulent flows over bluff-body geometries still represents a great challenge, RANS computations define a consolidated modelling approach for several industrial applications, whose ability in reproducing basic mean-flow features and turbulence statistics has been assessed over the last decades. The mean-flow analysis of Mettot, Sipp and Bézard ${ }^{27}$ based solely on the linearisation of the momentum and mass conservation equations is adopted here and applied to both the numerical and the experimental flow data, thus allowing a direct comparison of the obtained sensitivity maps. In the context of the adopted RANS modelling, we carefully investigate the impact of a frozen eddy viscosity approximation when introduced in the stability equations. Finally we show how the obtained stability results can be exploited to design an active feedback control of the unsteady wake targeting its vortex-shedding mechanism. The control design is based on the mean-flow linearised description of the fluid plant, under the well-established framework of the linear optimal control theory. ${ }^{4,5}$ The Minimal Energy Control (MCE) technique, ${ }^{29}$ which has been successfully applied to the laminar cylinder wake, ${ }^{30}$ is extended here to the control of organized low-frequency waves in turbulent flow oscillators, as described by the mean-flow global mode dynamics. The main advantage of this technique is that the computation of the stabilizing feedback gain field only requires the knowledge of the unstable adjoint mode, bypassing any model reduction step and thus providing directly a physical insight of the most relevant flow regions for a velocity feedback control.

The paper is organized as follows. We present in Sec. II the flow configuration, the employed numerical and experimental setups, and we compare the obtained results in terms of the vortex-shedding frequency and of the mean-wake flow structure. Then in Sec. III we introduce the global stability and sensitivity analyses of the computed and measured meanflows, with the details of the related numerical procedures. The proposed active feedback control of the unsteady RANS flow is described in Sec. IV, showing that the vortex-shedding is completely suppressed. In addition, a brief summary and concluding remarks are given in Sec. V.

\section{FLOW CONFIGURATION}

The considered flow configuration is illustrated in Fig. 1, featuring an elongated D-shaped cylinder of aspect ratio $A R=10$ located at the centre of a channel. The body leading edge is defined by a semi-ellipse which extends down to the mid-chord, while the remaining body half is represented by a thick flat-plate. The geometry in Fig. 1 exactly corresponds to a longitudinal plane of the wind-tunnel test section employed in our experimental setup, which is also illustrated in Fig. 3. Within this plane, the fluid motion is described using a Cartesian coordinate system where the $x$-axis is aligned with the direction of the incoming fluid stream, and the origin is located at the intersection between the symmetry axis and the body stern. The flow is modelled by means of the incompressible Navier-Stokes equations which are made dimensionless using the body thickness $H$, the velocity of the uniform fluid stream $U_{\infty}$ at the inlet and the constant density $\rho$. The corresponding Reynolds number is $R e=U_{\infty} H / v=32000, v$ being the kinematic viscosity of the fluid.

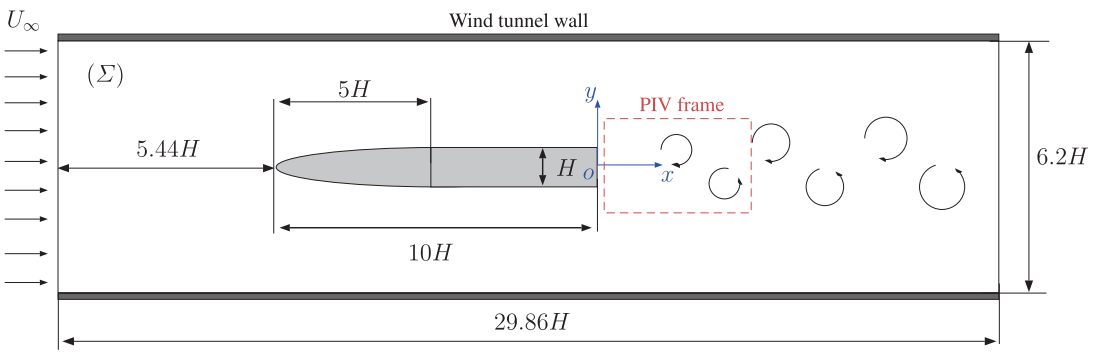

FIG. 1. Schematic diagram of the considered flow configuration. 


\section{A. 2D-RANS setup}

A two-dimensional RANS modelling is employed for the numerical simulation of the considered turbulent flow. Both steady and unsteady RANS computations have been performed using the OpenFOAM code. ${ }^{31}$ The implemented $k-\omega$ SST model $^{32}$ has been selected for such purpose, with the whole flow state being described by the velocity field $\boldsymbol{U}(x, y, t)$, the pressure field $P(x, y, t)$ and two additional scalar fields, $k(x, y, t)$ and $\omega(x, y, t)$ which are physically related to the turbulent kinetic energy and to the specific dissipation rate, respectively. The turbulent viscosity field $v_{t}(x, y, t)$ is defined as follows:

$$
v_{t}=\frac{a_{1} k}{\max \left(a_{1} \omega, \Omega\right)},
$$

where $a_{1}=0.31$ and $\Omega$ denotes the magnitude of the vorticity field. Within this framework the governing equations are spatially discretized using a finite volume approach, with a combination of centred and upwinded second-order discretization schemes. For time integration, a three-level secondorder backward difference method is employed. Given the incompressible description of the flow, the pressure-velocity coupling is handled using a standard "segregated" approach based on a momentum predictor, a pressure solver and a momentum corrector, exploiting the algorithms already implemented in OpenFOAM. In particular, the SIMPLE (semiimplicit method for pressure linked equations) $\operatorname{method}^{33}$ is used for steady-state RANS (S-RANS) computations while the PIMPLE method, which merges the SIMPLE and the PISO (pressure implicit with split operator) ${ }^{34}$ algorithms, has been used for the unsteady simulations. A unit streamwise velocity is imposed at the inlet of the computational domain $(\Sigma)$, where a turbulence level of $T u=2 \%$ and an eddy-tokinematic viscosity ratio of $v_{t} / v=100$ are prescribed, based on the recommendations from Menter, Kuntz and Langtry. ${ }^{35}$ On the outlet boundary, the governing equations are supplemented by homogeneous Neumann conditions for the velocity field, $\boldsymbol{\nabla} \boldsymbol{U} \cdot \hat{\boldsymbol{n}}=\mathbf{0}, \hat{\boldsymbol{n}}$ being the outward unit normal vector. Finally, consistently with the adopted near-wall mesh resolution, direct-wall boundary conditions ${ }^{36}$ are assigned on the solid surfaces:

$$
\boldsymbol{U}=\mathbf{0}, \quad k=0, \quad \omega=\frac{60 v}{\beta_{1} d^{2}},
$$

where $\beta_{1}=0.075$ and $d$ is the distance of the first cell centroid from the wall. For the pressure equation, homogeneous
Neumann conditions are assigned everywhere on the domain boundary except for the outflow boundary where the pressure is fixed to zero. The workflow is the following: first the S-RANS solution is computed using half of the mesh, thanks to the inherent mean-flow symmetry, and then it is employed as the initial condition for the U-RANS computations.

The employed mesh features a hybrid structure, as shown in Fig. 2. More precisely, the mesh is composed of a structured layer close to the wall boundaries (for both the body and the wind-tunnel surfaces) and of an unstructured region with triangular prismatic cells elsewhere. The use of hexahedral wall layers allows the better control of the near-wall mesh resolution and the accurate description of the turbulent boundary layers features. In particular, for the present computations, a strong clustering of the near-wall cells is adopted, with $d^{+} \leq 1$ everywhere, where the notation $(\cdot)^{+}$is used here and in the following to indicate the inner-wall scaling of the considered physical quantity. The total number of cells is $N_{c}=362162$.

In order to validate our numerical solver, we consider a well known benchmark represented by the flow past a square cylinder at $R e=U_{\infty} D / v=22000$, where $D$ is the length of the square side. For this test case, we employ the same computational box used by Bosch and Rodi, ${ }^{42}$ with a nearwall grid resolution fine enough to guarantee that $d^{+} \leq 1$ on the square boundary and resulting in a total mesh size of 43 200 cells. Similarly to the case of the thick flat-plate, a unit streamwise velocity is imposed at the inlet, where both $T u$ and $v_{t} / v$ are prescribed following the recommendations of the aforementioned authors. On the lateral boundaries, symmetry conditions are applied while at the outflow, homogeneous Neumann conditions hold for the velocity field. The dimensionless time-step is $\Delta t=2 \times 10^{-4}$. After a transient the flow settles down to a fully developed vortex-shedding regime, whereafter the solution is advanced in time and averaged on-the-fly over 600 time units (about 78 shedding cycles). Obtained results in terms of mean aerodynamic forces and nondimensional vortexshedding frequency $S t=f D / U_{\infty}$, are reported in Table I, and compare well with numerical and experimental data available from the literature.

\section{B. Experimental setup}

The experiments were conducted in an Eiffel wind tunnel at ONERA Toulouse. The test section, which is illustrated in

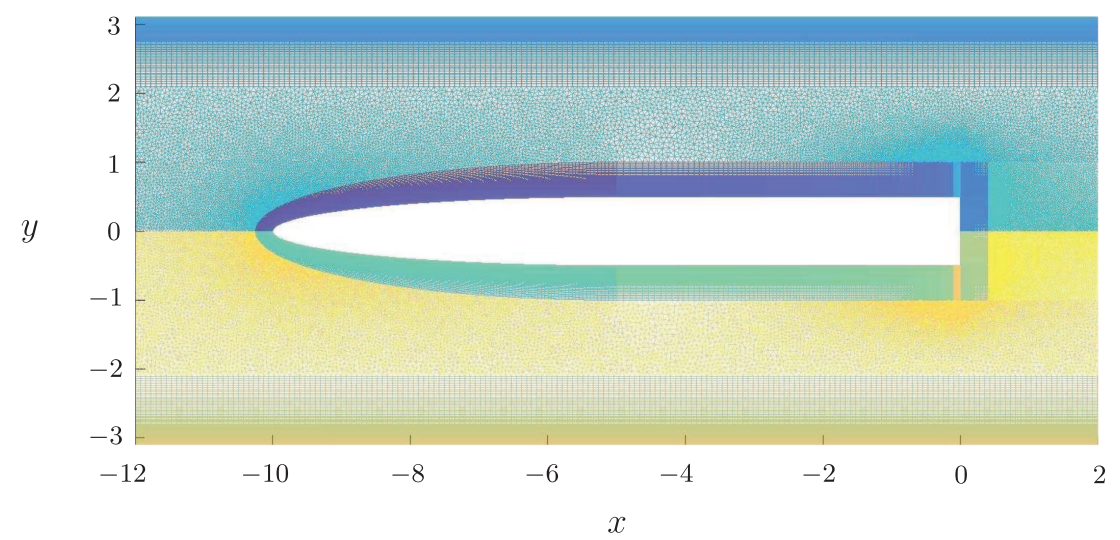

FIG. 2. Hybrid mesh employed for the RANS computations: detail of the mesh structure around the thick-flat plate. Colors are used to visualize the different structured and unstructured mesh regions. Note in particular the narrow mesh blocks introduced at the body blunt-edges to better capture the blowing/suction actuation; see Sec. IV for further details. 
TABLE I. Synoptic table of representative mean and fluctuating quantities for the flow past a square cylinder at $R e=22000 . \bar{C}_{D}$, denotes the mean drag coefficient, $C_{D}^{\prime}$ and $C_{L}^{\prime}$, the r.m.s. of the drag and lift coefficient signals and $S t$, the vortex-shedding Strouhal number.

\begin{tabular}{|c|c|c|c|c|c|}
\hline Contribution & Model & $\bar{C}_{D}$ & $C_{D}^{\prime}$ & $C_{L}^{\prime}$ & $S t$ \\
\hline Lyn et al. ${ }^{37}$ & Expt. & 2.1 & $\cdots$ & $\cdots$ & 0.132 \\
\hline $\mathrm{Lee}^{38}$ & Expt. & 2.05 & $0.16-0.23$ & $\ldots$ & $\ldots$ \\
\hline Rodi et $a l .{ }^{39}$ & LES & 2.2 & 0.14 & 1.01 & 0.13 \\
\hline Minguez et al..$^{40}$ & LES & 2.2 & $\cdots$ & $\ldots$ & 0.141 \\
\hline Rodi et al. ${ }^{39}$ & 2D RANS, two layer $k-\omega$ & 2.004 & $\ldots$ & $\ldots$ & 0.143 \\
\hline Iaccarino et al. ${ }^{41}$ & 2D RANS, $v^{2}-f$ & 2.22 & 0.056 & 1.83 & 0.141 \\
\hline Meliga et al..$^{25}$ & 2D RANS, SA & 2.26 & 0.37 & 1.13 & 0.139 \\
\hline Present & 2D RANS, $k-\omega$ & 2.14 & 0.27 & 1.58 & 0.13 \\
\hline
\end{tabular}

Fig. 3 has dimensions $1.2 \times 0.4 \times 0.31 \mathrm{~m}^{3}$ (length $\times$ width $\times$ height). The free-stream velocity ranges from $5 \mathrm{~m} / \mathrm{s}$ up to $50 \mathrm{~m} / \mathrm{s}$ with a measured turbulence level lower than $0.5 \%$. For the present experiments the free-stream velocity is set to $U_{\infty}=10 \mathrm{~m} / \mathrm{s}$, yielding the considered Reynolds number of $R e=32000$.

The D-shaped model is located at the mid-height of the test section with a zero angle of attack and spans over the entire width of the wind tunnel. The model is characterized by a chord length $\ell=500 \mathrm{~mm}$ and a trailing edge thickness $H=50 \mathrm{~mm}$, yielding a blockage ratio of $16.5 \%$. Given this bluff-body geometry, a wake observation area of length $\approx 10 \mathrm{H}$ was achieved. In order to force the boundary layer transition to the turbulent state at a fixed streamwise location, a $510 \mu \mathrm{m}$ zig-zag strip was placed at the $4.4 \%$ of the body chord starting from the leading-edge. Such a placement is motivated by the strong flow acceleration induced by the leading-edge geometry and the blockage ratio.

A high-speed stereo-PIV measurement system has been employed to characterize the wake of the thick-flat plate. After a preliminary verification of the two-dimensionality of the wake mean state with respect to the z-axis (see Fig. 3(a)), flow measurements were performed in the mid-span $x y$ plane of the model. The employed PIV system is composed of a Nd:YLF laser (Litron, LDY304 PIV, $527 \mathrm{~nm}, 30 \mathrm{~mJ}$ at $1 \mathrm{kHz}$ ) and two high-speed cameras (Phantom v711). The latter were mounted on Scheimpflug adapters positioned on each side of the light sheet and equipped with $105 \mathrm{~mm}$ lenses (AFD Micro Nikkor, $f 1: 2.8$ ) pointing to the measurement plane with an angle of $35^{\circ}$. The PIV measurements were performed at an acquisition frequency of $555 \mathrm{~Hz}$ over 5405 nondimensional time units. The velocity field and the related statistics were computed using the LaVision Davis 8.2 software, with final interrogation windows of $24 \times 24$ pixels and $75 \%$ overlap, which yield a PIV grid composed of 222 points in $x$ and of 122 points in $y$ for the rectangular box of coordinates $[0.20,4.46] \times[-1.12,1.21]$, which is represented in Fig. 1. The obtained spatial resolution is 0.963 $\mathrm{mm}$, corresponding to $\Delta x=\Delta y \approx 0.0193$ in nondimensional units.

\section{Results}

The computed RANS solutions of the confined flow past the thick flat-plate are depicted in Figs. 5 and 6 by means of the velocity field magnitude and of the turbulent viscosity field, respectively. A convergence study with respect to the employed near-wall mesh resolution has been performed based on the S-RANS results in order to reduce the required computational effort compared to the U-RANS case. Representative results are illustrated in Fig. 4, confirming that for $d^{+} \leq 1$, both the body and the wind-tunnel turbulent boundary layers are accurately captured. With reference to the employed mesh, the average and maximum values of $d^{+}$on the different wall boundaries are reported in Table II, indicating that the distribution of $d^{+}$does not significantly change when the timeaveraged U-RANS solution is considered. Note that averaging is performed over $\approx 30$ shedding cycles, as done in the study by Meliga et $a l .{ }^{43}$ for the turbulent flow past a square.

As expected, the S-RANS solution features a longer recirculation bubble compared to the U-RANS one, Figs. 5(a) and 5(c). At the same time, a remarkable difference is observed in the spatial distribution of the turbulent viscosity field. Indeed, while in the S-RANS case, Fig. $6(\mathrm{a}), \bar{v}_{t}(x, y)$ is mainly concentrated in the near-wake region, reaching its maximum (a)

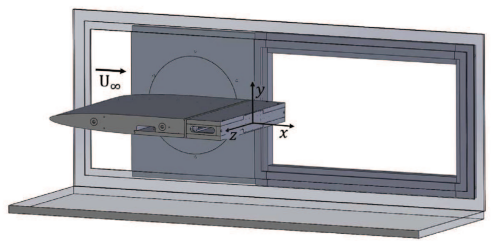

(b)

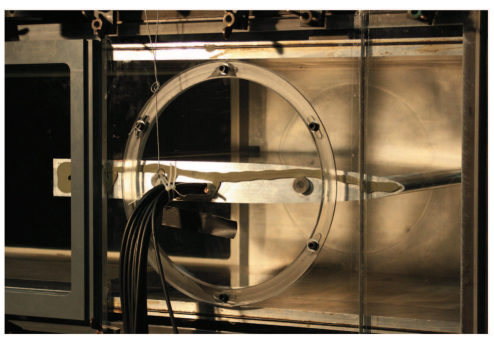

FIG. 3. Experimental setup. (a) Schematic of the D-shaped body installed in the wind tunnel test section. (b) Picture of the wind-tunnel test section. 
(a)

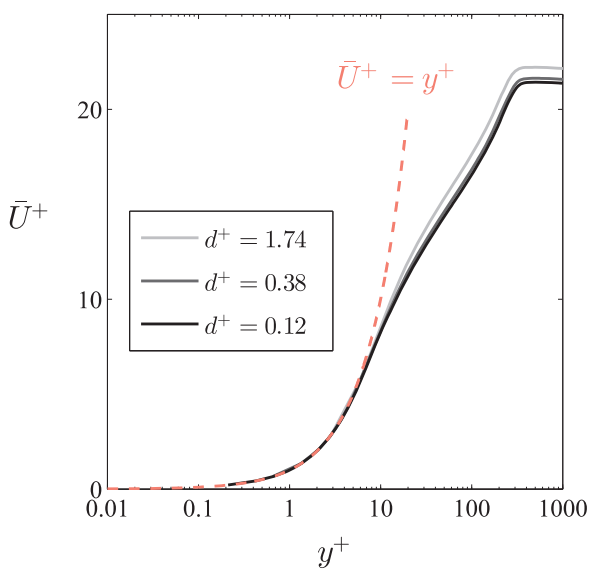

(b)

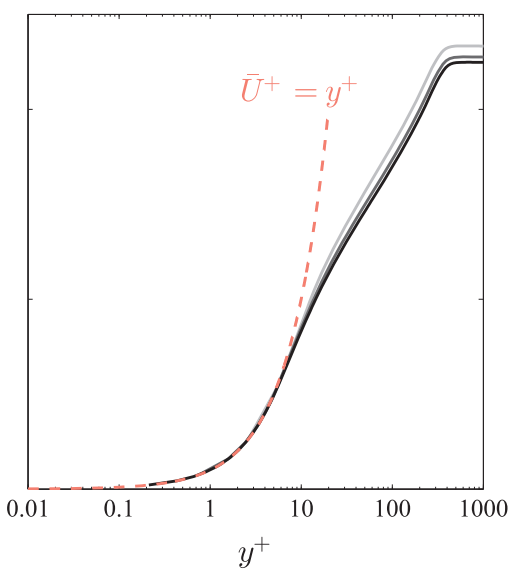

FIG. 4. Convergence study of the S-RANS mean flow in the near-wall region with respect to the adopted mesh resolution. (a) Thick-flat plate boundary layer at $x=-0.5$. (b) Wind tunnel boundary layer at $x=-0.5$.

TABLE II. Near-wall layer resolution at both the wind tunnel and the thick flat-plate solid surfaces. The average and the maximum values of the nondimensional distance of the first cell centroid from the wall, $d_{\text {avg }}^{+}$and $d_{\max }^{+}$, respectively, are reported.

\begin{tabular}{|c|c|c|c|c|c|c|}
\hline \multirow[b]{2}{*}{ Mean flow } & \multicolumn{2}{|c|}{ Wind tunnel wall } & \multicolumn{2}{|c|}{ Body wall $(x<0)$} & \multicolumn{2}{|c|}{ Body stern $(x=0)$} \\
\hline & $d_{\text {avg }}^{+}$ & $d_{\max }^{+}$ & $d_{\text {avg }}^{+}$ & $d_{\max }^{+}$ & $d_{\text {avg }}^{+}$ & $d_{\max }^{+}$ \\
\hline S-RANS & 0.36 & 1.0 & 0.30 & 0.40 & 0.1 & 0.30 \\
\hline U-RANS (time-averaged) & 0.37 & 1.0 & 0.35 & 0.82 & 0.3 & 0.55 \\
\hline
\end{tabular}

value just downstream of the recirculation bubble, in the timeaveraged U-RANS solution, Fig. 6(c), the eddy viscosity values gradually increase from the body stern up to the outflow boundary. Note that $(\bar{\cdot})$ is used to denote a time-averaged quantity. Similar considerations hold for $v_{t}(x, y, t)$ during the fully developed vortex-shedding regime, as shown by an instantaneous snapshot in Fig. 6(b), where the structure of the von Kármán wake is clearly highlighted. The corresponding velocity snapshot is depicted in Fig. 5(b). The flow evolution from the S-RANS state to the oscillatory regime is described in Figs. 7(a) and 7(c) by means of the time traces of the body aerodynamic coefficients which are defined based on the adopted reference velocity and length scales:

$$
C_{D}(t)=\frac{2 \boldsymbol{F}_{a}(t) \cdot \hat{\boldsymbol{x}}}{\rho H U_{\infty}^{2}}, \quad C_{L}(t)=\frac{2 \boldsymbol{F}_{a}(t) \cdot \hat{\boldsymbol{y}}}{\rho H U_{\infty}^{2}},
$$

where $\boldsymbol{F}_{a}(t)$ stands for the aerodynamic force, per unit width, acting on the thick flat-plate and $\hat{x}, \hat{\boldsymbol{y}}$ denote the unit vectors

(a)

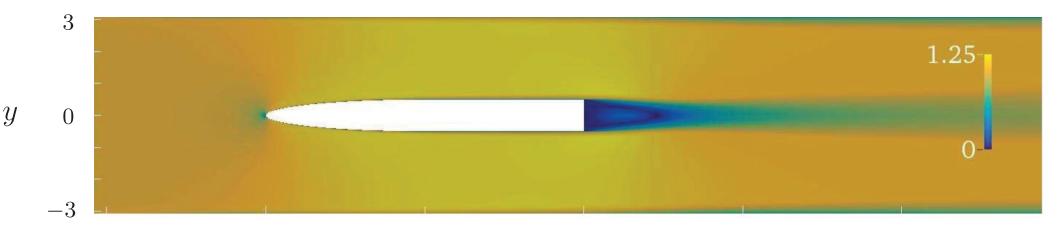

(b)

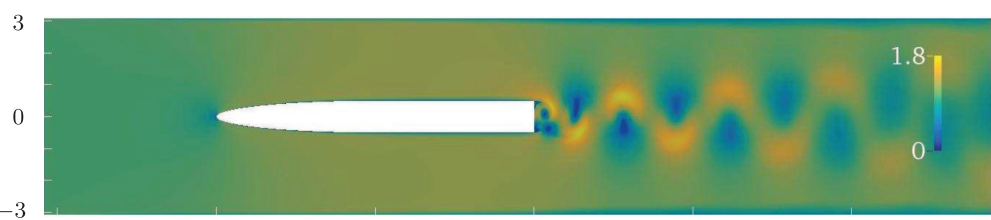

(c)

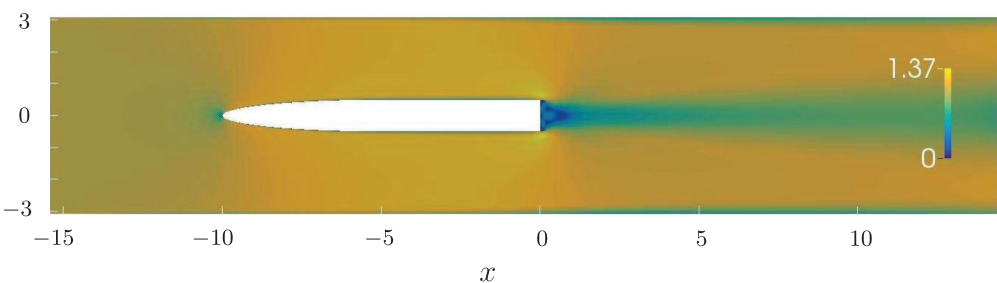

FIG. 5. RANS simulation of the confined flow past the thick flat-plate: magnitude of the velocity field, $\|\boldsymbol{U}\|$. (a) S-RANS solution. (b) U-RANS snapshot during the fully-developed vortexshedding regime. (c) Time-averaged U-RANS solution; averaging is performed over $\approx 30$ shedding cycles. 
(a)

(b)
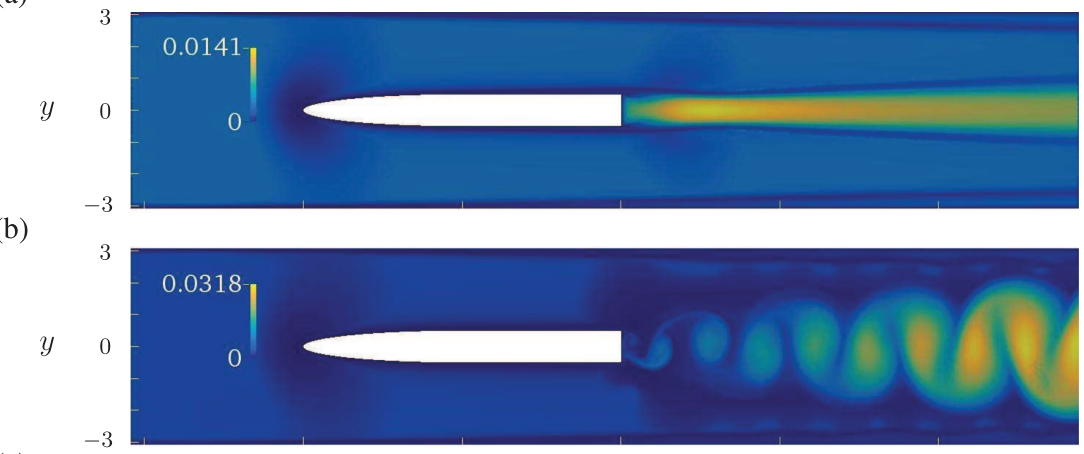

(c)

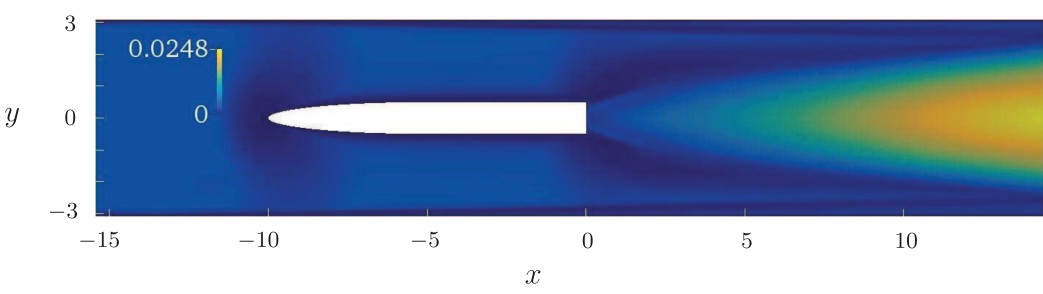

FIG. 6. RANS simulation of the confined flow past the thick flat-plate: turbulent viscosity field, $v_{t}$. (a) S-RANS solution. (b) U-RANS snapshot during the fully-developed vortex-shedding regime. (c) Time-averaged U-RANS solution; averaging is performed over $\approx 30$ shedding cycles. of the Cartesian reference system. At regime, the lift coefficient displays a fairly regular harmonic oscillation, Fig. 7(b), with a dominant frequency peak at $S t=f H / U_{\infty}=0.276$ and a small secondary peak at $S t=0.83$, which approximately corresponds to the third harmonic of the dominant frequency, Fig. 7(d). With the onset of the unsteady separation, the value of the mean drag coefficient $\bar{C}_{D}$ increases of $\approx 200 \%$. However, due to the high blockage ratio, care should be taken when comparing this value with experimental and numerical results aimed at characterizing similar geometries in an open-flow. By following the provisions of Barlow, Rae and Pope ${ }^{44}$ a first estimate of the effects introduced by the wind-tunnel walls for the considered two-dimensional geometry results in a corrected value of $\bar{C}_{D, c} \approx 0.87$, which appears more consistent with the value of $\approx 0.98$ measured by Pastoor et $a l .{ }^{45}$ for a D-shaped body of aspect-ratio 3.64 at $R e=23000$.

The obtained numerical results are then compared with the available PIV measurements of the turbulent wake. With reference to Table III, the time-averaged U-RANS solution underpredicts the recirculation length $L_{R}$, a fact that can be ascribed to the occurrence of strong three-dimensional phenomena (such as oblique vortex-shedding and vortex dislocations) in the near-wake dynamics. A value of $L_{R} \approx 0.85$ has been measured by Naghib-Lahouti, Lavoie and Hangan ${ }^{46}$ for a D-shaped body of $A R=12.5$, at $R e=30000$. At the same time, the estimated value of $S t$ results in close agreement with the experimental one. A small deviation is observed when (a)

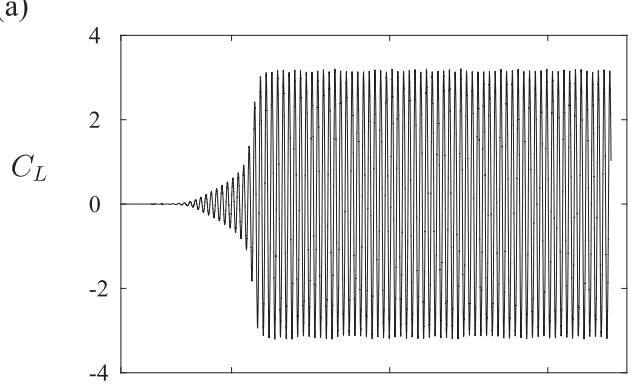

(c)

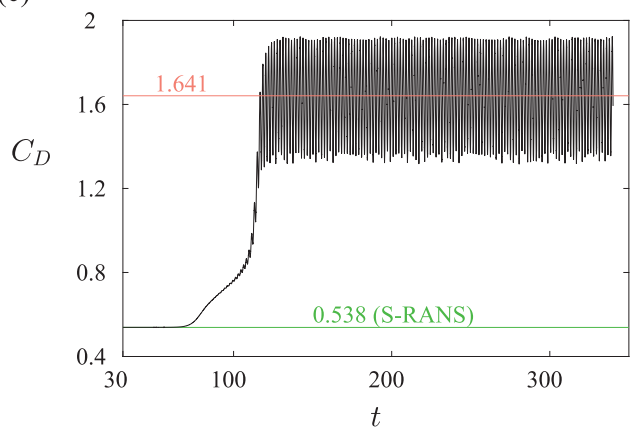

(b)

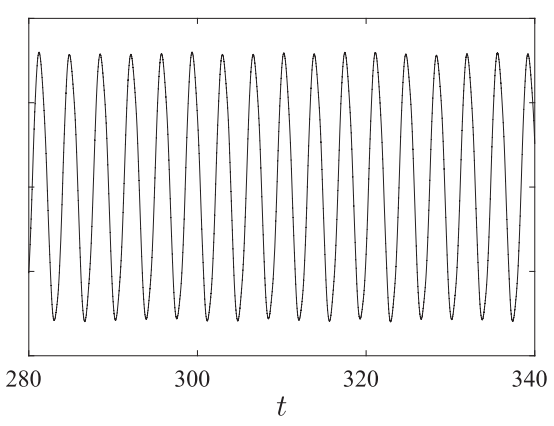

(d)

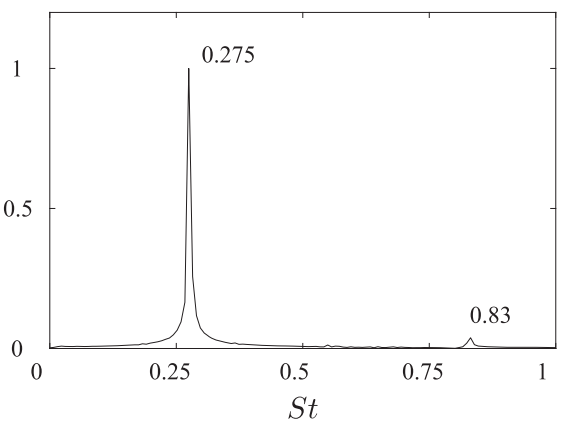

FIG. 7. Unsteady RANS simulation: time history of the thick-flat plate aerodynamic force coefficients. (a) Lift coefficient, $C_{L}$. (b) Detail view of $C_{L}$ when the vortex-shedding is fully developed. (c) Drag coefficient, $C_{D}$. (d) Spectral content of $C_{L}$ during the fully developed vortex-shedding regime. 
TABLE III. Confined turbulent flow past the thick flat-plate: Comparison between experimental and numerical results.

\begin{tabular}{lccc}
\hline \hline & $L_{R}$ & $S t$ & $S t_{e}$ \\
\hline U-RANS (time-averaged) & 0.67 & 0.275 & 0.207 \\
Expt. & 1.00 & 0.276 & 0.225 \\
\hline \hline
\end{tabular}

the vortex shedding frequency is made dimensionless using the effective body thickness $H_{e}=H+2 \delta^{*}$ (with $\delta^{*}$ being the boundary layer displacement thickness) and the maximum streamwise velocity $\bar{U}_{\max }$ at the trailing edge, thus accounting for the high blockage-ratio effects. The obtained values of $S t_{e, c}=f H_{e} / \bar{U}_{\max }$ are also listed in Table III and compare well with $S t_{e} \approx 0.231$ reported by the aforementioned authors in the range of $15000 \leq R e_{e} \leq 25000$, where $S t_{e}=f H_{e} / U_{\infty}$ and $R e_{e}=U_{\infty} H_{e} / v$, and with the value of $S t_{e} \approx 0.229$ obtained by Bull et $a l .{ }^{47}$ for blunt trailing-edge profiled bodies with fully turbulent boundary layers. Finally, a detailed comparison of the near-wake flow structure is illustrated in Fig. 8 by inspecting the streamwise velocity profile at different $x$ stations downstream of the body stern. The major deviations from the experimental measurements are observed just downstream of the flow separation, where the detached shear-layers display an excessive diffusion with respect to the experimental measurements. On the contrary, moving downstream, the turbulent wake is better approximated in its self-similar region. The fact that the numerical results cannot adequately capture the details of the reverse flow region (as frequently occurs for RANS computations around bluff-body geometries) could question the physical relevance of their subsequent stability and sensitivity analyses. However, in the aforementioned work, ${ }^{25}$ Meliga, Pujals and Serre have shown that, even if $L_{R}$ is underestimated by $\approx 30 \%$ (as in the present case), the global stability analysis still provides a fairly good prediction of the measured vortex-shedding frequency, and, moreover, a very good qualitative agreement in terms of the experimental frequency sensitivity map obtained by Parezanović and Cadot. ${ }^{26}$ The comparison between the experimental and numerical mean flow could probably be improved by means of more advanced and computationally expensive techniques, such as LES or DES, which are, however, still far from the state-of-the-art of many industrial CFD applications.

\section{GLOBAL STABILITY AND SENSITIVITY ANALYSES}

The global stability and sensitivity analyses of the considered turbulent flow are carried out based on the so-called mean flow approach, as described by Mettot, Sipp and Bézard. ${ }^{27}$ This approach simply relies on the linearisation of the mass conservation and momentum equations around the given timeaveraged mean flow, either numerically computed or experimentally measured, neglecting any turbulence modelling. Although lacking of a rigorous mathematical foundation, past studies $^{18,19,25,27}$ have shown that the mean-flow linearised Navier-Stokes equations can be used to efficiently predict the leading frequency of large-scale organized waves in various laminar and turbulent flows driven by an oscillator-like instability mechanism. A common variant of this approach is based on the use of a modified viscosity $\tilde{v}(x, y)$ in the stability equations, with $\tilde{v}$ being equal to the sum of the molecular viscosity $v$ and of the time-averaged eddy viscosity $\bar{v}_{t}(x, y)$. This variant, often known as frozen eddy-viscosity approach, is also employed here in the analysis of the U-RANS data, investigating the impact of such an approximation on the stability and sensitivity results. In the following, the same terminology introduced by Mettot, Sipp and Bézard ${ }^{27}$ will be adopted, with the mean-flow approach based on the molecular viscosity only being termed quasi-laminar approach, and its frozen eddyviscosity variant, quasi-laminar mixed approach. Finally, the global stability of the S-RANS solution will be also examined using the above described methodologies. However, it is worthwhile to note that, in this case, the stability analysis should be interpreted within a base-flow like framework, ${ }^{19,27}$ since the involved mean flow, which corresponds to a steady solution of the RANS equations, is only driven by the Reynolds stresses stemming from the fine-scale turbulent motions and not by the large-scale flow unsteadiness. The relevance of such results for the active control of the flow will be addressed in Sec. IV. (a)

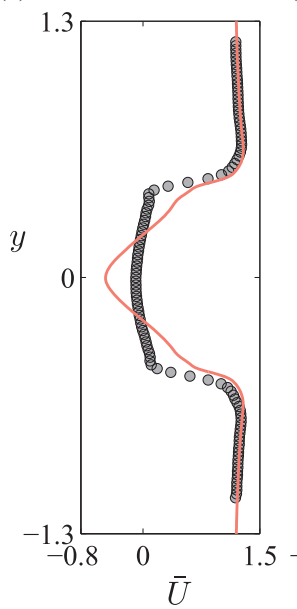

(b)

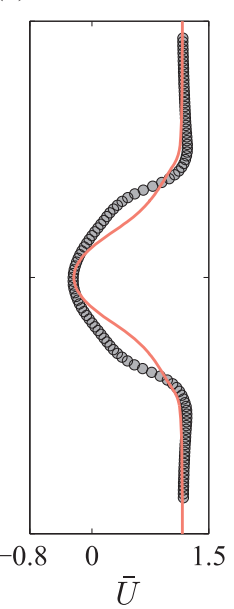

(c)

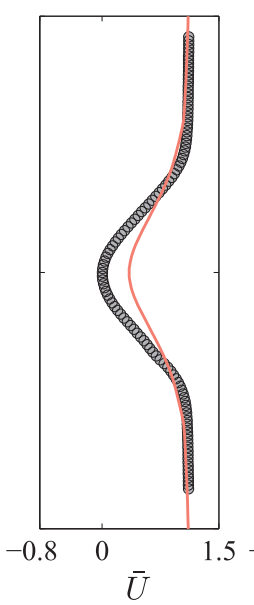

(d)

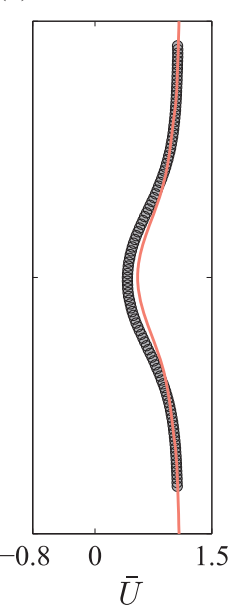

(e)

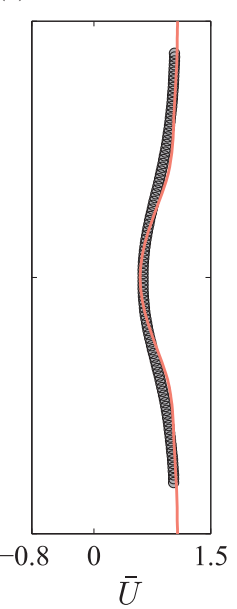

FIG. 8. Mean flow past the thick flatplate: near-wake velocity profiles at different $x$ stations. Comparison between the U-RANS prediction (continuous red line) and the PIV experimental measurements (grey dots). (a) $x=0.1974$. (b) $x=0.5057$. (c) $x=1.007$. (d) $x=1.5076$. (e) $x=2.0028$. 


\section{A. Mathematical formulation}

The linear global stability analysis of the given mean flow $Q_{m}(x, y)=\left(U_{m}, P_{m}\right)$ is performed by solving for a small perturbation field $\boldsymbol{q}=(\boldsymbol{u}, p)$ in the normal mode form $\boldsymbol{q}(x, y, t)$ $=\hat{q}(x, y) \exp (\lambda t)$, where $\hat{q}(x, y)$ denotes the spatial mode shape and $\lambda \in \mathbb{C}$. By introducing this ansatz into the "linearised" incompressible Navier-Stokes equations around $\boldsymbol{Q}_{m}$, we get

$$
\begin{aligned}
\lambda \hat{\boldsymbol{u}}+\left(\boldsymbol{U}_{m} \cdot \boldsymbol{\nabla}\right) \hat{\mathbf{u}}+(\hat{\mathbf{u}} \cdot \boldsymbol{\nabla}) \boldsymbol{U}_{m} & \\
+\boldsymbol{\nabla} \hat{p}-\boldsymbol{\nabla} \cdot\left(\tilde{v}\left(\boldsymbol{\nabla} \hat{\mathbf{u}}+\boldsymbol{\nabla} \hat{\mathbf{u}}^{T}\right)\right) & =\mathbf{0}, \\
\boldsymbol{\nabla} \cdot \hat{\mathbf{u}} & =0,
\end{aligned}
$$

where $\tilde{v}(x, y)=1 / R e+\bar{v}_{t}(x, y)$ and $(\cdot)^{T}$ stands for the transpose. Note that any term stemming from the linearisation of the turbulence model has been neglected in the above formulation, except for the frozen turbulent diffusion. When the quasi-laminar approach is employed $\left(\bar{v}_{t}=0\right)$, this corresponds to implicitly assume that, at least at a first-order, the turbulence affects the dynamics of the large-scale fluctuations only indirectly, through the induced mean-flow corrections. As concerns the boundary conditions, a distinction has to be made between the analysis of the numerical and the experimental data. In the first case, the same computational domain employed for the RANS computations is adopted, where $\hat{\boldsymbol{u}}$ is assumed to vanish at the inlet and on the solid boundaries while the following condition is imposed at the outflow:

$$
\hat{p} \hat{\boldsymbol{n}}-\tilde{v}\left(\boldsymbol{\nabla} \hat{\boldsymbol{u}}+\boldsymbol{\nabla} \hat{\boldsymbol{u}}^{T}\right) \cdot \hat{\boldsymbol{n}}=\mathbf{0} .
$$

In the stability analysis of the experimental data, which are available only within a smaller flow region (see Fig. 1), the above condition, Eq. (5), is imposed on the whole boundary of the corresponding domain, except at the inlet, where $\hat{\boldsymbol{u}}=\mathbf{0}$. Once supplemented with these homogeneous boundary conditions, Eq. (4) define a generalized eigenvalue problem for $\lambda$. Any solution $\hat{\boldsymbol{q}}$ associated with an eigenvalue $\lambda$ represents a global mode of the mean-flow linearised dynamics with growth-rate $\mathfrak{R}(\lambda)$ and angular frequency $\mathfrak{J}(\lambda)$. The sensitivity properties of the leading global mode are then investigated by computing and making use of the properties of the corresponding adjoint mode, $\hat{\boldsymbol{q}}^{\dagger}=\left(\hat{\boldsymbol{u}}^{\dagger}, \hat{p}^{\dagger}\right)$, which is solution of the following (adjoint) eigenvalue problem:

$$
\begin{aligned}
\lambda^{*} \hat{\mathbf{u}}^{\dagger}-\left(\boldsymbol{U}_{m} \cdot \boldsymbol{\nabla}\right) \hat{\mathbf{u}}^{\dagger} & \\
+\boldsymbol{\nabla} \boldsymbol{U}_{m}{ }^{T} \cdot \hat{\mathbf{u}}^{\dagger}+\nabla \hat{p}^{\dagger}-\boldsymbol{\nabla} \cdot\left(\tilde{v}\left(\boldsymbol{\nabla} \hat{\mathbf{u}}^{\dagger}+\nabla \hat{\mathbf{u}}^{\dagger}\right)\right) & =\mathbf{0} \\
\boldsymbol{\nabla} \cdot \hat{\mathbf{u}}^{\dagger} & =0
\end{aligned}
$$

where $(\cdot)^{*}$ stands for the complex conjugate. As discussed by Giannetti and Luchini ${ }^{10}$ and by Pralits, Brandt and Giannetti, ${ }^{11}$ the regions of the flow acting as a "wavemaker" in the excitation of the global instability mechanism can be identified as the regions of highest receptivity to a structural perturbation in the form of a local force-velocity feedback,

$$
\lambda^{\prime} \mathcal{E}(x, y) \hat{\boldsymbol{q}}^{\prime}-\mathcal{A}(x, y) \hat{\boldsymbol{q}}^{\prime}=\mathcal{H}\left(x, y ; x_{0}, y_{0}\right) \hat{\boldsymbol{q}}^{\prime},
$$

where we have introduced the following compact notation for the linearised Navier-Stokes operator

$$
\mathcal{E}(x, y) \boldsymbol{q}=\left(\begin{array}{c}
\boldsymbol{u} \\
0
\end{array}\right), \quad \mathcal{A}(x, y) \boldsymbol{q}=\left(\begin{array}{c}
-\left(\boldsymbol{U}_{m} \cdot \boldsymbol{\nabla}\right) \boldsymbol{u}-(\boldsymbol{u} \cdot \boldsymbol{\nabla}) \boldsymbol{U}_{m}-\boldsymbol{\nabla} p+\boldsymbol{\nabla} \cdot\left(\tilde{v}\left(\boldsymbol{\nabla} \boldsymbol{u}+\boldsymbol{\nabla} \boldsymbol{u}^{T}\right)\right) \\
\boldsymbol{\nabla} \cdot \boldsymbol{u}
\end{array}\right)
$$

and the feedback operator

$$
\mathcal{H}\left(x, y ; x_{0}, y_{0}\right) \boldsymbol{q}=\left(\begin{array}{c}
\delta\left(x-x_{0}, y-y_{0}\right) \mathcal{K}_{0} \boldsymbol{u} \\
0
\end{array}\right),
$$

$\mathcal{K}_{0}$ being a constant feedback tensor while $(\cdot)^{\prime}$ has been used to denote the perturbed quantities. By carrying out a firstorder sensitivity analysis of the perturbed eigenvalue problem, Eq. (7), the corresponding first-order variation of $\lambda, \delta \lambda$, can be expressed as follows:

$$
\delta \lambda=\mathcal{S}\left(x_{0}, y_{0}\right): \mathcal{K}_{0}
$$

where $\mathcal{S}(x, y)$ is the structural sensitivity tensor associated with the considered global mode and defined as

$$
\mathcal{S}(x, y)=\frac{\left(\hat{\boldsymbol{u}}^{\dagger}\right)^{*} \otimes \hat{\boldsymbol{u}}}{\left\langle\hat{\boldsymbol{u}}^{\dagger}, \hat{\boldsymbol{u}}\right\rangle}
$$

with

$$
\left\langle\hat{\boldsymbol{u}}^{\dagger}, \hat{\boldsymbol{u}}\right\rangle=\int_{\Sigma}\left(\hat{\boldsymbol{u}}^{\dagger}\right)^{*} \cdot \hat{\boldsymbol{u}} d \Sigma
$$

being the vector-field scalar product on the considered spatial domain $(\Sigma)$. In Eqs. (10) and (11), the symbols ":" and " $\otimes$ " stand for the double contraction and the dyadic products, respectively. Relevant information about the eigenvalue sensitivity can be extracted by plotting at each spatial point a suitable norm of $\mathcal{S}(x, y)$, such as, for instance, its Frobenious norm, $\|\mathcal{S}(x, y)\|_{F}$, for which the following identity holds:

$$
\|\mathcal{S}(x, y)\|_{F}=\left\|\hat{\boldsymbol{u}}^{\dagger}(x, y)\right\|\|\hat{\boldsymbol{u}}(x, y)\|,
$$

with the global modes being normalized such that $\left\langle\hat{\boldsymbol{u}}^{\dagger}, \hat{\boldsymbol{u}}\right\rangle=1$.

In addition to the wavemaker analysis, the eigenvalue sensitivity to a perturbation of the mean flow defines another important quantity to get a physical insight in the instability mechanism, especially for control purpose. Indeed it provides a useful tool to predict how the mean flow should be varied by the control action in order to significantly affect the leading global mode. In particular, this kind of analysis has been shown to accurately predict the vortex-shedding frequency variation produced by a small secondary cylinder placed in the turbulent wake of a D-shaped body. ${ }^{25,27}$ When a generic variation of the considered mean flow $\delta \boldsymbol{U}_{m}$ is assumed, at a first-order, 
the corresponding eigenvalue drift can be formally expressed as

$$
\delta \lambda=\left\langle\boldsymbol{\nabla}_{\boldsymbol{U}_{m}} \lambda, \delta \boldsymbol{U}_{m}\right\rangle,
$$

where the complex-valued sensitivity vector-field $\boldsymbol{\nabla}_{\boldsymbol{U}_{m}} \lambda$ has the following expression ${ }^{11,17}$

$$
\nabla_{\boldsymbol{U}_{m}} \lambda=-\nabla \hat{\boldsymbol{u}}^{H} \cdot \hat{\boldsymbol{u}}^{\dagger}+\left(\hat{\boldsymbol{u}}^{*} \cdot \nabla\right) \hat{\boldsymbol{u}}^{\dagger},
$$

with $(\cdot)^{H}$ indicating Hermitian conjugation.

\section{B. Numerical method}

The linear stability and sensitivity problems are numerically solved by means of a finite element discretization on unstructured grids made of triangular cells, using a standard $\mathbb{P}_{2}-\mathbb{P}_{1}$ representation for the velocity and the pressure perturbation fields, respectively. The open-source finite element library FEniCS ${ }^{48}$ is employed for such purpose. Within this numerical framework all the required matrix inversions are handled by means of the sparse direct-solver MUMPS ${ }^{49,50}$ while the involved large-scale eigenvalue problems are solved using the Krylov-Schur algorithm implemented in the SLEPc library, ${ }^{51,52}$ with a shift-invert transformation. A "discrete" approach is adopted to compute the adjoint modes, which allows one to account for the proper boundary conditions of the adjoint problem automatically and to preserve the bi-orthogonality property of the eigenmodes up to machine precision.

Once computed using the RANS solver, both the velocity and the turbulent viscosity fields are linearly interpolated from the original hybrid mesh to a triangularization of the same computational domain made of 834470 triangles, which correspond to a total number of 3774800 degrees of freedom. A strong mesh refinement is introduced in the neighbourhood of the thick flat-plate blunt edges with a minimum mesh size of $\approx 5 \times 10^{-5}$, to properly capture the extremely localized features of the adjoint mode, as will be described in the following. At the same time, in order to reduce the computational effort without significantly affecting the stability results, the unstructured grid is made a little bit coarser in the wind-tunnel boundary layer regions which will be shown to marginally contribute to the stability and sensitivity spatial structures. A mesh refinement with a subsequent linear interpolation of the turbulent mean flow is also introduced in the stability analysis of the experimental data. Starting from the PIV measurement window, the computational domain is resized in the $y$ direction to $[-0.85,0.85]$, in order to skip those regions where the available flow measurements are not trustable. Then, the initial triangle mesh is easily obtained from the original Cartesian PIV grid, resulting in a total number of 35024 triangles which is increased up to 537347 by means of subsequent refinement steps, avoiding the introduction of any numerical stabilization term of the governing equations.

\section{Results}

\section{Global modes}

The global spectrum extracted from the analysis of the RANS mean flow is illustrated in Fig. 9, for both the S-RANS and the time-averaged U-RANS flow fields, Figs. 9(a) and 9(b), respectively. In both cases, the spectrum computed using the quasi-laminar approach (black round dots) features the existence of a leading unstable mode whose frequency is close to the vortex-shedding frequency. In addition, and in analogy to the results described by Mettot, Sipp and Bézard, ${ }^{27}$ we observe the existence of some slightly unstable modes lying on the real axis. When the turbulent diffusion is taken into account in the stability analysis (quasi-laminar mixed approach), the whole spectrum is damped, with all the aforementioned real modes becoming stable. Moreover, the estimation of the vortex-shedding frequency is further improved. In Fig. 9(b) the quasi-laminar spectrum of the PIV measured mean-flow is also reported. The structure of the spectrum is analogous to the one observed for the U-RANS based results, although the mode displaying the vortex-shedding frequency is much more damped. It is interesting to note that no unstable real modes have been observed in the global eigenspectrum reported by Camarri, Fallenius and Fransson ${ }^{28}$ for the PIV measured mean-flow past a porous cylinder at $R e=3500$.

The spatial structures of the direct and adjoint modes are compared in Fig. 10 for the PIV based and the U-RANS based quasi-laminar computations. The direct mode, represented by its real streamwise component in Figs. 10(a) and 10 (c), displays the typical pattern of the von Kármán instability, and despite a phase difference still remains after adopting the same normalization, the two mode shapes compare fairly well. Note that the computational domain employed for the stability analysis of the experimental mean flow is exactly the one used to depict the direct mode in Fig. 10(c). (a)

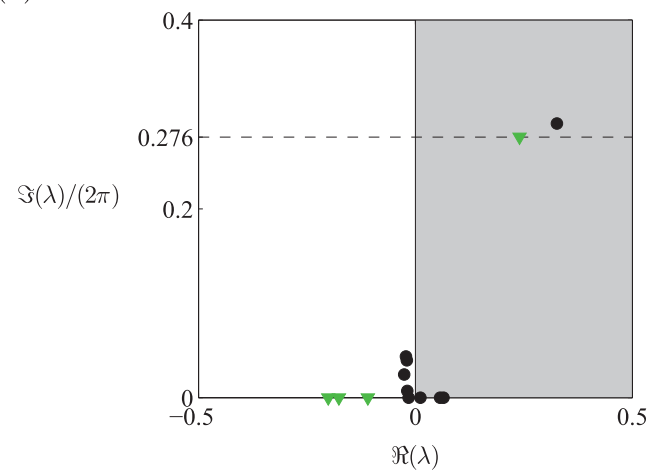

(b)

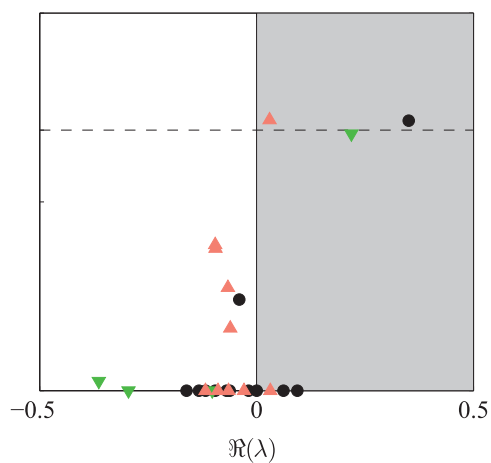

FIG. 9. Global eigenspectrum of the turbulent mean flow past the thick flat-plate. (a) S-RANS mean flow. (b) U-RANS (time averaged) mean flow. For both cases, results obtained using the quasi-laminar approach (black dots) and the quasi-laminar mixed approach (green downward triangles) are illustrated. In the panel (b) the spectrum extracted from the quasi-laminar stability analysis of the PIV measured mean flow is also reported (upward red triangles). 
(a)

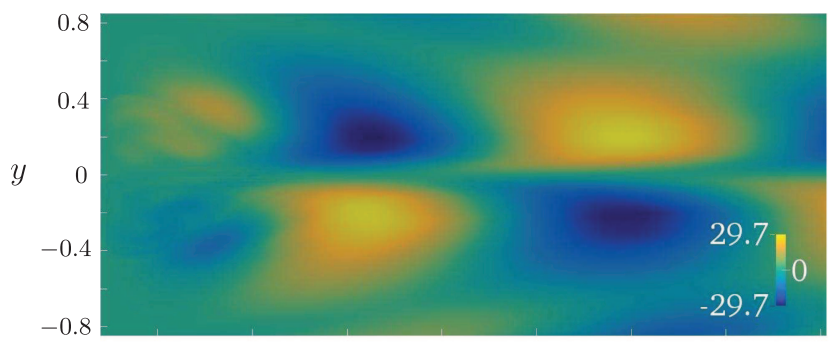

(c)

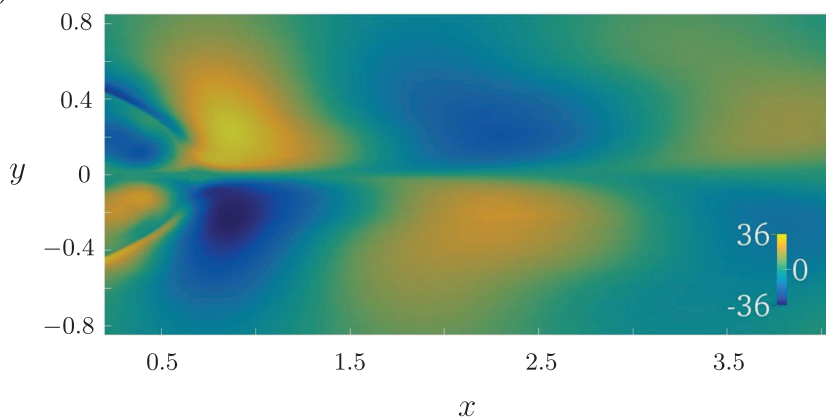

The spatial distribution of the adjoint field magnitude is illustrated in Figs. 10(b) and 10(d). In this case, each scalar map has been rescaled to the maximum value attained within the employed representation window. Notwithstanding the difference in the near-wake structure, both modes are highly localized on the boundary of the recirculation bubble, showing qualitatively the same spatial distribution. As already mentioned, in order to accurately capture the thin layers characterizing the adjoint mode, the original PIV mesh has been strongly refined in the near-wake region. The computed eigenvalue for an increasing mesh resolution is reported in Table IV. In addition, similarly to what has been done by Camarri, Fallenius and Fransson, ${ }^{28}$ the influence of the adopted boundary conditions and of the continuity errors are investigated. When the velocity disturbances are assumed to vanish on the lateral boundaries, the eigenvalue associated with the vortexshedding mode moves to the left-half of the complex plane and the associated frequency increases of $\approx 10 \%$, thus indicating that these boundary conditions are too severe for the small employed computational domain. On the contrary, when the experimental mean flow is projected on a divergence-free (b)

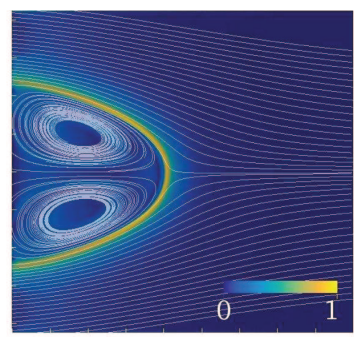

(d)

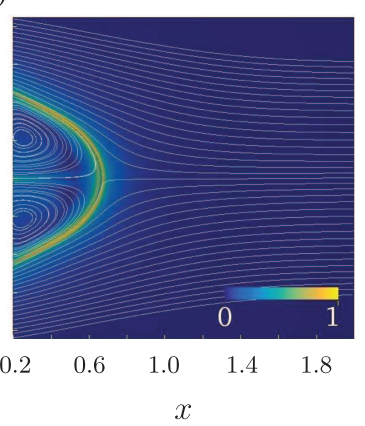

FIG. 10. Leading direct and adjoint global modes resulting from the stability analysis of the PIV measured ((a) and (b)) and the U-RANS computed ((c) and (d)) time-averaged mean flows. For both cases the quasi-laminar approach is used. ((a) and (c)) Real streamwise component of the direct mode velocity field normalized using the condition $\hat{u}(2,0)=1$. ((b) and (d)) Magnitude of the adjoint mode velocity field normalized with respect to its maximum value within the considered region. In the panels ((b) and (d)) the mean flow streamlines are also illustrated.

subspace at a pre-processing stage, only a slight variation of the eigenvalue is observed. More precisely, the projection step is performed by solving the following system of coupled equations:

$$
\begin{aligned}
\boldsymbol{U}_{m}^{c} & =\boldsymbol{U}_{m}-\boldsymbol{\nabla} \phi, \\
\boldsymbol{\nabla} \cdot \boldsymbol{U}_{m}{ }^{c} & =0,
\end{aligned}
$$

where $\boldsymbol{U}_{m}^{c}$ is the corrected mean flow and the scalar field $\phi(x, y)$ is a Lagrange multiplier introduced to enforce the incompressibility constraint. The above system is discretized using the finite element method outlined in Sec. III B and $\phi$ is computed by solving the associated Schur-complement system, thus avoiding the introduction of ad hoc boundary conditions for $\phi$. It is interesting to note that homogeneous Dirichlet conditions on the lateral boundaries have been employed by Camarri, Fallenius and Fransson ${ }^{28}$ without observing any damping effect on the leading eigenvalue, although the crosswise extent of their computational domain is comparable to the present one. However, a slight sensitivity of the eigenvalue to these boundary conditions is shortly mentioned by the authors.

TABLE IV. Quasi-laminar stability analysis of the PIV measured mean flow past the thick flat-plate. Eigenvalue associated with the vortex-shedding instability obtained for different mesh resolutions and boundary conditions on the top and bottom sides of the computational box. The results obtained by projecting the experimental mean flow on a divergence-free subspace are also reported.

\begin{tabular}{lccrr}
\hline \hline$N_{e}$ & Bcs top \& bottom side & Divergence free & $\mathfrak{R}(\lambda) \times 10^{2}$ & $\mathfrak{J}(\lambda)$ \\
\hline 35024 & Stress-free & No & 3.028394 & 1.801678 \\
70839 & Stress-free & No & 2.989337 & 1.801810 \\
123441 & Stress-free & No & 2.987804 & 1.801801 \\
181191 & Stress-free & No & 2.985676 & 1.801798 \\
461943 & Stress-free & No & 2.985290 & 1.801795 \\
537347 & Stress-free & No & 2.985263 & 1.801795 \\
537347 & Homogeneous Dirichlet & No & -6.178652 & 1.981686 \\
537347 & Stress-free & Yes & 4.060024 & 1.778353 \\
\hline \hline
\end{tabular}


(a)

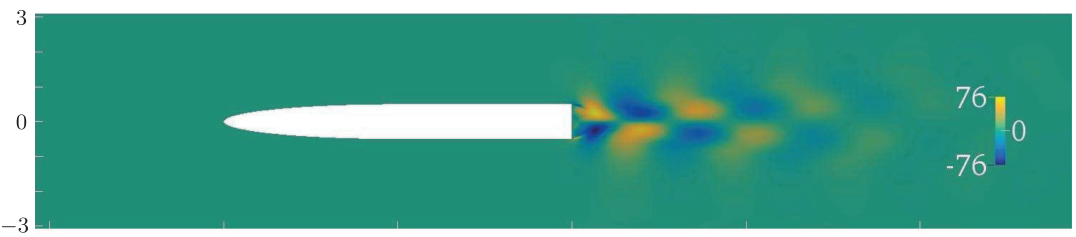

(b)

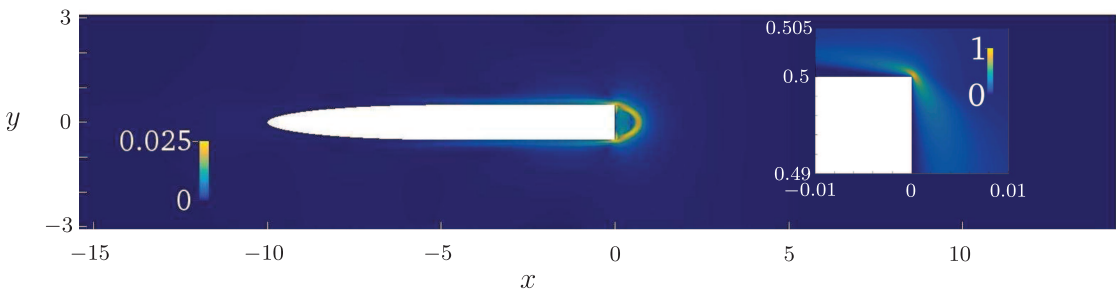

FIG. 11. Leading direct and adjoint global modes resulting from the stability analysis of the time-averaged U-RANS mean flow using the quasi-laminar mixed approach. (a) Real streamwise component of the direct-mode velocity field normalized using the condition $\hat{u}(2,0)=1$. (b) Magnitude of the adjoint-mode velocity normalized with respect to its maximum value. In the panel (b) a color-scale saturated at the $2.5 \%$ has been employed to better highlight the overall spatial structure of the adjoint mode.
For the sake of comparison, the above representations of the direct and adjoint modes have been necessarily limited to the small measurement window. Their whole spatial structure as extracted from the quasi-laminar mixed analysis of the U-RANS mean flow is depicted in Fig. 11. By comparing Figs. 10(c) and 11(a), we can observe that the turbulent diffusion does not substantially modify the shape of the direct mode. On the contrary, the sharp gradients characterizing the adjoint mode are noticeable smoothed, Figs. 10(d) and 11(b). Note that, with respect to Fig. 10(d), the adjoint map illustrated in Fig. 11(b) has now been rescaled to the maximum value attained over the entire computational domain $(\Sigma)$. However, in the same picture, a colormap saturated at $2.5 \%$ has been used for visualization purposes, in order to better highlight the regions of highest receptivity in the near-wake. Indeed, the inspection of the whole spatial structure of the adjoint mode reveals that the mode maxima are extremely localized at the blunt edges of the thick flat-plate, as shown in the inset of Fig. 11(b) (where a full-range colormap is used instead), with an amplitude of two orders of magnitude greater than the values reached on the boundary of the recirculation bubble. The same considerations hold also for the quasi-laminar results where the difference in amplitude with the thinner layers of nearwake receptivity reduces to $\approx 1$ order of magnitude, as well as for the S-RANS-based results (not shown here). As already mentioned, an ad hoc mesh refinement has been introduced to accurately capture this fine-scale structure of the adjoint mode, and, as an example, the convergence study carried out for the S-RANS-based quasi-laminar results is summarized in Table V.

Note that similar features of the adjoint mode have not been described, nor pointed out in past studies of the global

TABLE V. Quasi-laminar stability analysis of the computed S-RANS mean flow past the thick flat-plate. Leading eigenvalue convergence with respect to an increasing mesh resolution. Successive mesh refinement areas have been introduced around the thick flat-plate blunt edges to properly capture the adjoint global mode maxima.

\begin{tabular}{lcc}
\hline \hline$N_{e}$ & $\mathfrak{R}(\lambda)$ & $\mathfrak{I}(\lambda)$ \\
\hline 737447 & 0.325948 & 1.823954 \\
778411 & 0.326169 & 1.824223 \\
873011 & 0.326194 & 1.824223 \\
\hline
\end{tabular}

stability properties of turbulent flow past a D-shaped cylinder. $^{25,27}$ The existence of a receptivity pocket at the sharp corner where flow separation occurs (and its implications for flow control) have been described not only in several studies concerning cavity flows, ${ }^{53-55}$ but also for a backward facing step geometry. ${ }^{56}$ Despite, the experimental reverse mean flow is poorly captured by the numerical results, the detached upper and lower boundary layer profiles reported in Fig. 8(a) are well reproduced, which can suggest that also the detaching boundary layer at the trailing-edge is adequately captured. Unfortunately, no PIV measurements were performed in this region, which prevented us to include it in the stability analysis of the experimental mean flow to get a direct comparison with the above results. As it will be shown in the following, these receptivity pockets are suggested to play a relevant role in the sensitivity picture as well as in the active control of the flow.

\section{Sensitivity analysis}

In agreement with the theory shortly recalled in Sec. III A, we can now quantify the unstable mode sensitivity to a forcevelocity feedback, Eq. (11), and its sensitivity to a generic perturbation of the mean flow, Eq. (15). The corresponding maps obtained from the quasi-laminar analysis of the numerical (U-RANS) and the experimental time-averaged mean flows are compared in Fig. 12. Similarly to the adjoint mode depicted in Figs. 10(b) and 10(d), the regions of highest sensitivity are localized along the shear-layers delimiting the average recirculation bubble, and more precisely, towards the closure of the bubble itself. The structure of both the wavemaker in Fig. 12(a) and of the frequency sensitivity field, $\mathfrak{I}\left(\boldsymbol{\nabla}_{\boldsymbol{U}_{m}} \lambda\right)$, shown in Figs. 12(d) and 12(e), closely resemble the ones described for the turbulent flow past a porous cylinder. ${ }^{28}$ However the maximum sensitivity is remarkably higher in the present case, of one order of magnitude for $\|\mathcal{S}(x, y)\|_{F}$ and of two orders of magnitude for $\mathfrak{T}\left(\nabla_{\boldsymbol{U}_{m}} \lambda\right)$. The U-RANS and the experimental based results agree well, both in terms of the sensitivity spatial distribution with respect to the underlying mean flow structure, and of the attained values. The main difference is represented by the existence of a secondary region of high sensitivity across the wake centreline, which is not observed in the PIV based results. The existence of this additional region can be associated with the higher back-flow velocity observed in the computed mean-flow, which is approximately two times greater than the corresponding experimental value. 
(a)

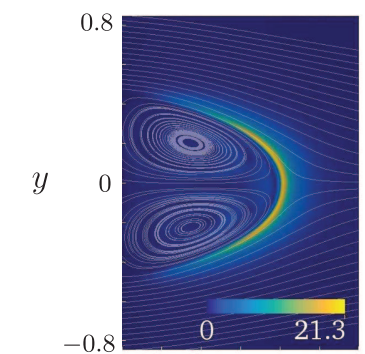

(f)

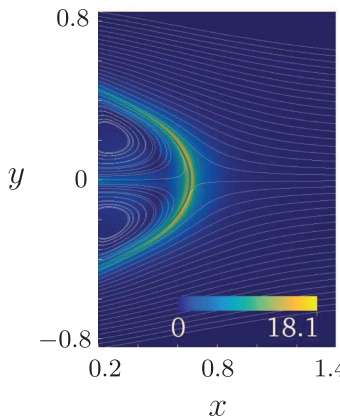

(b)

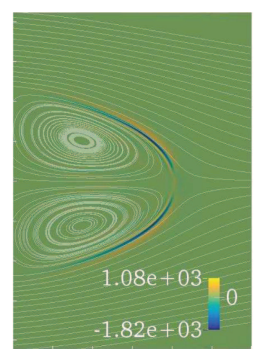

(g)
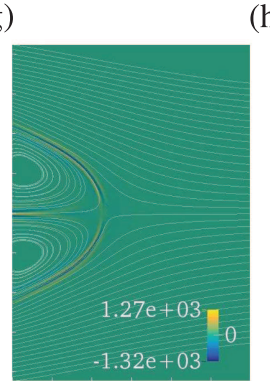

(c)

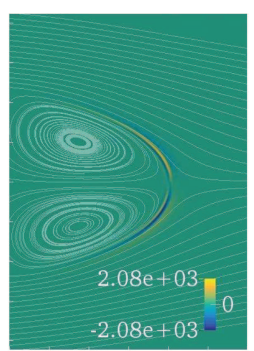

(h)

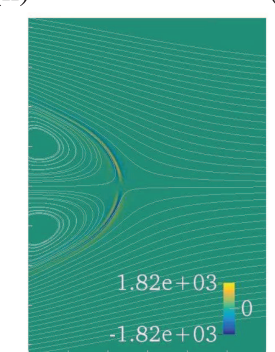

(d)

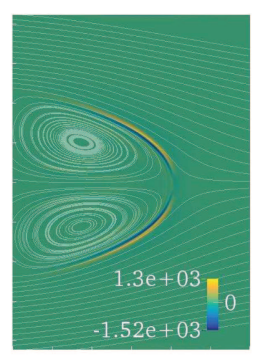

(i)

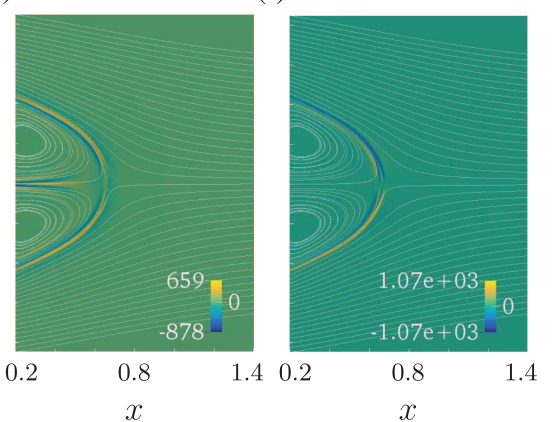

FIG. 12. Quasi-laminar sensitivity analysis of the unstable global mode associated with the vortex-shedding instability. ((a)-(e)) PIV based results. ((f)-(l)) U-RANS based results. ((a) and (f)) Sensitivity to a local force-velocity feedback. ((b) and (g)) Mode growth-rate sensitivity with respect to streamwise meanflow modifications, $\mathfrak{R}\left(\nabla_{U_{m}} \lambda\right) \cdot \hat{\boldsymbol{x}}$. ((c) and (h)) Mode growth-rate sensitivity with respect to crosswise mean-flow modifications, $\mathfrak{R}\left(\boldsymbol{\nabla}_{U_{m}} \lambda\right) \cdot \hat{\boldsymbol{y}}$. ((d) and (i)) Mode frequency sensitivity with respect to streamwise mean-flow modifications $\mathfrak{J}\left(\nabla_{U_{m}} \lambda\right) \cdot \hat{\boldsymbol{x}}$. ((e) and (1)) Mode frequency sensitivity with respect to crosswise mean-flow modifications, $\mathfrak{J}\left(\nabla_{U_{m}} \lambda\right) \cdot \hat{\boldsymbol{y}}$. Note that in the bottom panels the employed colormaps are saturated at the range of values attained within the represented spatial region.

(a)

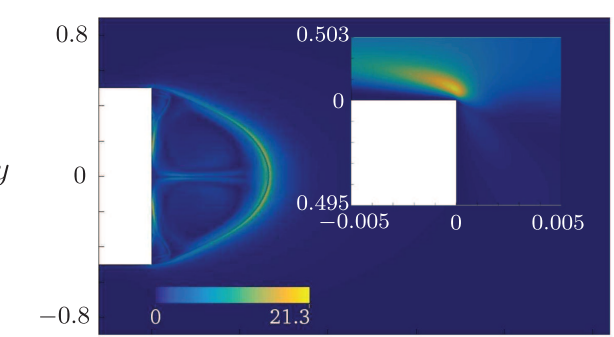

(c)

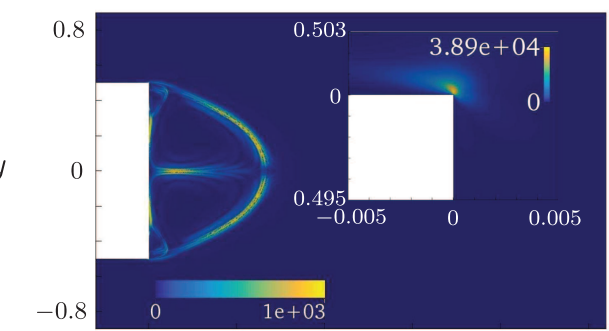

(e)

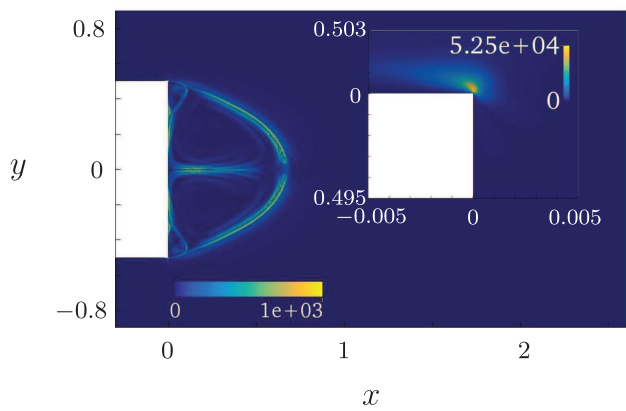

(b)

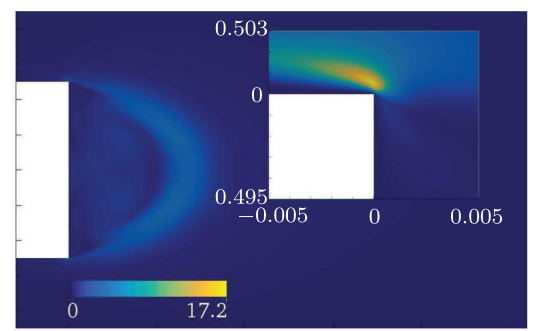

(d)

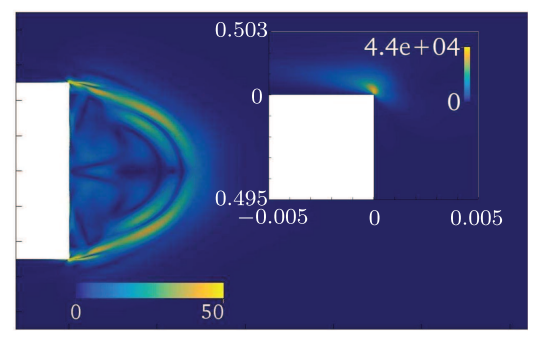

(f)

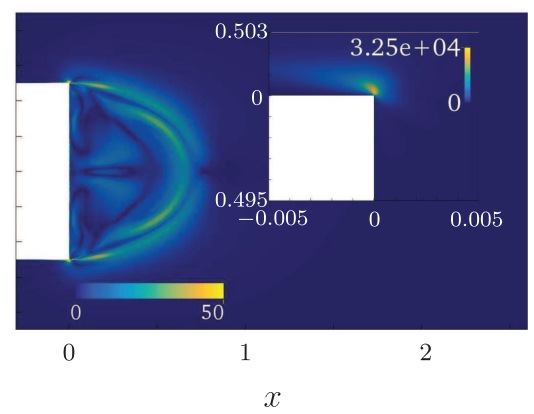

FIG. 13. Adjoint-based sensitivity analysis of the leading unstable mode associated with the time-averaged U-RANS mean flow. Left column: quasi-laminar approach. Right column: quasi-laminar mixed approach. ((a) and (b)) Sensitivity to a local force-velocity feedback. ((d) and (e)) Magnitude of the mode growth-rate sensitivity with respect to mean-flow modifications, $\left\|\mathfrak{R}\left(\nabla_{U_{m}} \lambda\right)\right\|$. ((f) and (g)) Magnitude of the mode frequency sensitivity with respect to mean-flow modifications, $\left\|\mathfrak{I}\left(\nabla_{U_{m}} \lambda\right)\right\|$. Note that in the central and bottom row panels saturated colormaps have been employed while the corresponding full-range colormap is reported in the corresponding insets. 
The effects of the additional turbulent diffusion on the mean-flow sensitivity are investigated in Fig. 13 by comparing the quasi laminar and the quasi-laminar mixed results obtained from the analysis of the time-averaged U-RANS mean flow. In both cases the visual inspection of the sensitivity maps, which now include the body stern region, shows that the highest sensitivity is always attained within a tiny pocket just above the blunt edge of the thick-flat plate. This is true also for the wavemaker structure in Figs. 13(a) and 13(b), thus suggesting (according to the analysis of Giannetti and Luchini ${ }^{10}$ ) that the "core" of the instability is composed of two distinct regions: a primary region corresponding to the blunt edge, and a secondary one at the end of the recirculation bubble, already described in Figs. 12(a) and 12(f). This is even more evident when the turbulent viscosity is taken into account in the sensitivity analysis, Fig. 13(b), with the values of $\|\mathcal{S}\|_{F}$ close to the body stern being approximately one order of magnitude greater than those found at the downstream edge of the recirculation bubble. The difference between these two regions further increases when considering the scalar maps associated with $\left\|\mathfrak{R}\left(\boldsymbol{\nabla}_{\boldsymbol{U}_{m}} \lambda\right)\right\|$ and $\left\|\mathfrak{I}\left(\boldsymbol{\nabla}_{\boldsymbol{U}_{m}} \lambda\right)\right\|$, depicted in Figs. 13(c)-13(f), respectively. Indeed, due to the additional turbulent diffusion, the sensitivity layers lying on the boundary of the recirculation bubble are considerably smoothed, while the thin region along the centreline almost disappears. At the same time, it is worthwhile to note that the shape of the blunt-edge sensitivity pocket is not substantially affected by $\bar{v}_{t}$, and although the corresponding peak value is different between the quasi laminar and the quasi-laminar mixed results, it keeps the same order of magnitude.

\section{ADJOINT-BASED FEEDBACK CONTROL}

The mean-flow stability analysis has been shown to accurately capture the leading frequency of the unsteady thick flatplate wake, especially when a quasi-laminar mixed approach is used. In this section we investigate the possibility to further exploit these results in order to actively control the global instability of the considered flow. Recent studies ${ }^{25,27}$ have shown how the mean-flow based sensitivity analysis can be used to effectively predict before hand the impact of a small control cylinder on the vortex-shedding frequency of a bluffbody wake, thus paving the way for the efficient design of passive control devices. Motivated by these results, we numerically explore here the possibility to design an active feedback control of the considered flow based on the linearized NavierStokes equations around a selected mean-flow, leaving aside any turbulence modelling except for the frozen eddy viscosity approximation, as done in the stability and sensitivity analyses. This makes the control design independent of the particular choice of the turbulence model. Indeed, in a real control experiment, while we can have access to phase-averaged measurements of the turbulent flow field, it is rather difficult or even unrealistic to adequately estimate turbulence modelling quantities, such as $k(x, y, t)$ and $\omega(x, y, t)$, even based on their physical counterparts.

In the following we consider a full-information control within the well-established framework of the linear optimal control theory. ${ }^{4,5,57}$ More precisely, we present here an original extension of the Minimal Control Energy (MCE) technique $^{29,30}$ to the control of turbulent bluff-body wakes. This technique allows the efficient computation of a stabilizing feedback rule of the linearised flow model based solely on the knowledge of the unstable adjoint modes. The proposed extension relies on the particular choice of the mean flow employed to build up the linear model which corresponds to the S-RANS solution instead of the time-averaged U-RANS one. It is well known that for bluff-body configurations, the steady solution of the RANS equations often exhibits a lower mean drag coefficient with respect to the real one, due to the overprediction of the mean wake recirculation length. Indeed, both these quantities are better estimated by means of unsteady RANS computations, where the mean-flow corrections due to the large-scale instabilities are taken into account. Nevertheless the quasi-laminar mixed analysis performed around the S-RANS mean flow has shown that the vortex-shedding frequency is accurately predicted, Fig. 9(a). These observations provide us the rational to employ the S-RANS mean flow in the derivation of the linearised flow model for control design. Indeed, as shown in the control of the laminar cylinder wake, ${ }^{30}$ the base solution around which the "linearisation" is performed, i.e., the generalized base flow, not only specifies the linearised dynamics, but also plays the role of the "target flow" of the control action. Therefore, a control design based on the S-RANS mean flow is expected to drive the unsteady flow around a time-averaged state characterized by an elongated mean wake with a lower pressure drag.

Finally, even if it would be hard to introduce a fullinformation feedback control in a real control experiment (since it would require the measurement, at each time instant, of the whole flow field), the inspection of the spatial distribution of the computed feedback gain (vector) field can provide relevant information about those flow regions which are of utmost importance for feedback control, thus guiding and supporting the experimental investigations. In addition, bypassing any open-loop model reduction, it is possible to assess the best control performance in absence of model reduction errors, with the model reduction step being postponed after the control design step, i.e., in closed loop, or restricted to the estimation problem only.

\section{A. Control definition, methodology and implementation}

The unsteady RANS flow is controlled by means of a couple of blowing/suction slots symmetrically positioned on the upper and lower surfaces of the thick flat-plate for $x \in[-0.1,-0.05]$, as sketched in Fig. 14. This placement of the actuators, very close to the body stern results as a compromise between the need of a realistic control configuration, with body-embedded actuators, and the results of the sensitivity analysis described in Sec. III C 2, showing that the highest sensitivity is located at the blunt edge of the thick flat-plate. The reference crosswise velocity profile of the blowing/suction actuation is also illustrated in the inset of Fig. 14, featuring a smoothed top-hat shape which is given by the following expression:

$v(\xi)=h_{m}(\xi / w-1)+h_{m}((\xi-1) / w+1), \quad \xi \in[0,1]$, 


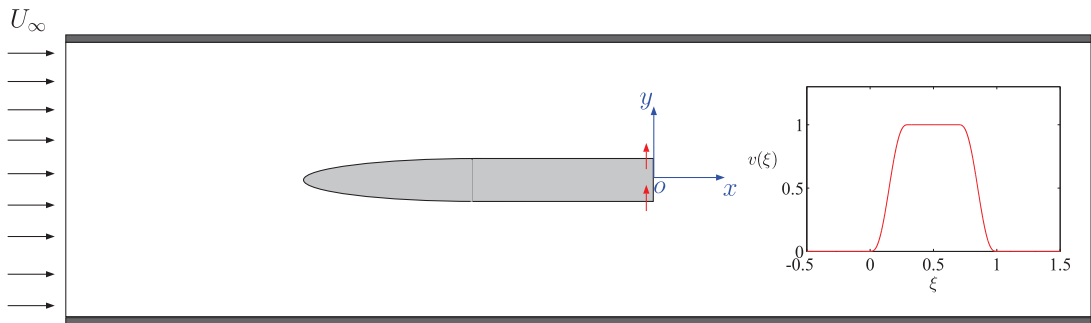

FIG. 14. Schematic diagram of the flow configuration with active feedback control. Blowing/suction slots (denoted by the small red arrows) are introduced on the upper and lower body surfaces close to the stern, for $x \in[-0.1,-0.05]$. The inset illustrates the non-dimensional reference jet profile for the $y$ velocity component.

where $\xi$ is a non-dimensional abscissa along the slot width and $h_{m}(s)$ is a mollified step function defined as

$$
h_{m}(s)=\left\{\begin{array}{lll}
0 & \text { if } s \leq-1, \\
0.5+s\left(0.9375-s^{2}\left(0.625-0.1875 s^{2}\right)\right) & \text { if } s \in(-1,1), \\
1 & \text { if } s \geq 1,
\end{array}\right.
$$

where the smoothing parameter $w$ has been set equal to $w=0.15$. Note that the two actuators are operated in phase opposition with the same amplitude, resulting in a zero netmass blowing/suction actuation. Therefore, a single control variable $\varphi(t)$ is defined, which represents the instantaneous ${ }^{58}$ maximum blowing/suction velocity, where a positive sign means blowing from the upper surface and suction from the lower one.

Given the above configuration and the linearised description of the flow dynamics around the considered mean flow, the "flow plant", is defined as follows:

$$
\begin{aligned}
& \mathcal{E}(x, y) \frac{\partial \boldsymbol{q}}{\partial t}=\mathcal{A}(x, y) \boldsymbol{q}, \quad \text { on }(\Sigma), \\
& \left.\boldsymbol{q}(x, y, t)\right|_{\Gamma_{c}}=\boldsymbol{h}(x, y) \varphi(t), \\
& \left.\mathcal{B}(x, y) \boldsymbol{q}(x, y, t)\right|_{\partial \Sigma / \Gamma_{c}}=\mathbf{0},
\end{aligned}
$$

where $h(x, y)$ stands for the spatial distribution of the applied Dirichlet condition on the control boundary $\Gamma_{c}$. On the remaining portion of $(\partial \Sigma)$ the same homogeneous boundary conditions of the eigenvalue problem (4), here formally expressed through the operator $\mathcal{B}(x, y)$, are applied. The above boundary control problem can be conveniently recast in a volume control formulation by means of a lifting procedure ${ }^{6}$ of the inhomogeneous datum on $\left(\Gamma_{c}\right)$. This is obtained by introducing the solution $\boldsymbol{q}_{c}(x, y)$ of the following steady problem:

$$
\begin{aligned}
& \mathcal{A}(x, y, t) \boldsymbol{q}_{c}=\mathbf{0}, \quad \text { on }(\Sigma), \\
& \left.\boldsymbol{q}_{c}(x, y)\right|_{\Gamma_{c}}=\boldsymbol{h}(x, y), \\
& \left.\mathcal{B}(x, y) \boldsymbol{q}_{c}(x, y)\right|_{\partial \Sigma / \Gamma_{c}}=\mathbf{0},
\end{aligned}
$$

and then expressing the original flow state as $\boldsymbol{q}(x, y, t)$ $=\tilde{\boldsymbol{q}}(x, y, t)+\boldsymbol{q}_{c}(x, y) \varphi(t)$ where, upon a substitution into Eq. (19), we derive the following problem for the auxiliary flow state $\tilde{\boldsymbol{q}}(x, y, t)$ :

$$
\begin{aligned}
& \mathcal{E}(x, y) \frac{\partial \tilde{\boldsymbol{q}}}{\partial t}=\mathcal{A}(x, y) \tilde{\boldsymbol{q}}+\boldsymbol{b}(x, y) c(t), \quad \text { on }(\Sigma), \\
& \left.\tilde{\boldsymbol{q}}(x, y, t)\right|_{\Gamma_{c}}=\mathbf{0}, \\
& \left.\mathcal{B}(x, y) \tilde{\boldsymbol{q}}(x, y, t)\right|_{\partial \Sigma / \Gamma_{c}}=\mathbf{0},
\end{aligned}
$$

where $\boldsymbol{b}(x, y)=\mathcal{E}(x, y) \boldsymbol{q}_{c}(x, y)$ and $c(t)=-d \varphi(t) / d t$. The above equations are spatially discretized using the same finite element approach described in Sec. III B, obtaining

$$
E \frac{d x}{d t}=A x(t)+B c(t)
$$

with $\boldsymbol{x}(t)$ being the array of velocity and pressure states, while the matrices $E, A$ and $B$ denote the discrete representation of the linear operators $\mathcal{E}(x, y), \mathcal{A}(x, y)$ and of the forcing vector field $\boldsymbol{b}(x, y)$, respectively. At this point, the linear feedback control is designed directly within the discrete setting, with a control law of the form $c(t)=K \boldsymbol{x}(t)$. More precisely, the feedback gain (row) matrix $K$ is computed as the MCE solution of the classical Linear Quadratic Regulator (LQR) problem, ${ }^{57}$ through the following formula: ${ }^{30,59}$

$$
K=-r^{-1} B_{u}^{H} F^{-1} P_{u}^{H} E,
$$

where $P_{u}$ is the matrix of the unstable left eigenvectors of the pencil $(A, E)$, i.e., the discrete representation of the complexconjugate pairs of unstable adjoint modes, $\hat{\boldsymbol{q}}^{\dagger}$ and $\left(\hat{\boldsymbol{q}}^{\dagger}\right)^{*}$, $B_{u}=P_{u}^{H} B$ and $r$ is the control weight associated with the definition of the optimal control cost function $J=\int_{0}^{\infty} x^{H} Q x$ $+r c(t)^{2} d t$ which is minimized. Note that the state weight matrix $Q$ does not enter the computation of the MCE solution. ${ }^{59}$ Furthermore, it can be shown that when a single control variable is considered, the particular value of $r$, with $r \geq 0$, does not affect the resulting gain values. ${ }^{30}$ In the following expressions, $r$ is reported only for the sake of completeness and we can simply assume $r=1$. The matrix $F$ in Eq. (23) is defined as

$$
F=\left[\begin{array}{cc}
\frac{M_{11}}{2 \mathfrak{R}(\lambda)} & \frac{M_{12}}{2 \lambda} \\
\frac{M_{21}}{2 \lambda} & \frac{M_{22}}{2 \mathfrak{R}(\lambda)}
\end{array}\right] \text { with } \quad M=r^{-1} B_{u} B_{u}^{H} .
$$

Therefore, as previously anticipated, the computation of the MCE feedback gains requires only the knowledge of the unstable adjoint modes of the the mean-flow linearised dynamics. Once computed, the linear feedback rule can be re-interpreted within the continuous setting by introducing the field $\boldsymbol{q}_{k}(x, y)$ of feedback gains: 
TABLE VI. MCE control law: computed values of $\alpha, \tilde{\alpha}$ and $\beta$ for both the laminar cylinder wake at $R e=50$ and the turbulent flow past the thick flat-plate at $R e=32000$.

\begin{tabular}{lccc}
\hline \hline & $\alpha$ & $\tilde{\alpha}$ & $\beta$ \\
\hline Laminar cylinder wake & $-6.3871 \times 10^{-2}$ & $-7.6871 \times 10^{-2}$ & $-6.8107 \times 10^{-14}$ \\
Turbulent thick-flat plate flow & $-9.6076 \times 10^{-1}$ & -1.2010 & $-3.2720 \times 10^{-12}$ \\
\hline \hline
\end{tabular}

$$
c(t)=K \boldsymbol{x}(t)=\boldsymbol{k}^{H} E \boldsymbol{x} \approx \int_{\Sigma} \boldsymbol{q}_{k}^{*} \cdot \mathcal{E}(x, y) \tilde{\boldsymbol{q}} d \Omega=\left\langle\boldsymbol{u}_{k}, \tilde{\boldsymbol{u}}\right\rangle,
$$

where the array $\boldsymbol{k}=-r^{-1} P_{u} F^{-H} B_{u}$ denotes the discrete counterpart of $\boldsymbol{q}_{k}(x, y)=\left(\boldsymbol{u}_{k}, p_{k}\right)$, storing the corresponding velocity and pressure gain degrees of freedom. It is worthwhile to observe that in the derived feedback law, the pressure does not contribute to $c(t)$, since $E$ is positive semidefinite, according to the incompressibility constraint. Both $\boldsymbol{k}$ and $\boldsymbol{q}_{k}$ are realvalued quantities and, in the present case, simply correspond to a linear combination of the real and the imaginary parts of the unstable adjoint mode. We can further manipulate Eq. (25) in order to express the feedback law in terms of the original control variable $\varphi(t)$ and of the inhomogeneous linearised flow state $\boldsymbol{q}(x, y, t)$. By taking into account the lifting procedure and substituting for the definition of $\tilde{\boldsymbol{q}}(x, y, t)$ and $c(t)$ in Eq. (25), we get

$$
\frac{d \varphi(t)}{d t}=-\left\langle\boldsymbol{u}_{k}, \boldsymbol{u}-\varphi(t) \boldsymbol{u}_{c}\right\rangle=\alpha \varphi(t)-\left\langle\boldsymbol{u}_{k}, \boldsymbol{u}\right\rangle
$$

with $\alpha=\left\langle\boldsymbol{u}_{k}, \boldsymbol{u}_{c}\right\rangle$ being a constant coefficient. An additional step is required when the control is applied directly to the nonlinear RANS flow model. In fact, consistently with the adopted linear mean-flow model, within the fully nonlinear U-RANS setting $\boldsymbol{q}$ is approximated as the difference between the instantaneous flow velocity $\boldsymbol{U}(x, y, t)$ and the considered mean-flow $\boldsymbol{U}_{m}(x, y)$, yielding to

$$
\frac{d \varphi(t)}{d t}=\alpha \varphi(t)-\left\langle\boldsymbol{u}_{k}, \boldsymbol{U}\right\rangle+\beta,
$$

where the additional constant coefficient $\beta$ is equal to $\beta=\left\langle\boldsymbol{u}_{k}, \boldsymbol{U}_{m}\right\rangle$. It is worthwhile to note that, due to the introduced lifting procedure, a feedback control of integral type is finally obtained in terms of $\varphi(t)$

$$
\varphi(t)=\varphi(0) e^{\alpha t}-\int_{0}^{t} e^{\alpha(t-\tau)} \gamma(\tau) d \tau
$$

with $\gamma(t)=\left\langle\boldsymbol{u}_{k}, \boldsymbol{U}\right\rangle-\beta$. Indeed it is easy to show that the overall closed-loop system features a pole at the origin, which can lead to a constant value of $\varphi(t)$ different from zero at infinite time, i.e., a constant non zero actuation. In order to prevent this, at least within the linearised setting, the value of $\alpha$ can be tuned by a small variation which results to be approximately of the same order of $-\mathfrak{R}(\lambda)$, and in the following we denote by $\tilde{\alpha}$, the modified value of $\alpha$ which is employed in the simulations of the control systems.

Within the adopted U-RANS setup described in Sec. II A, the feedback control is numerical implemented using a "segregated-like" approach where at each time-step of the closed-loop dynamics, the value of $\varphi(t)$ (which enters the boundary conditions of the U-RANS equations) is linearly extrapolated from the previous time-steps and, at the same time, the coupling term $\left\langle\boldsymbol{u}_{k}, \mathbf{U}\right\rangle$ in Eq. (27) is integrated explicitly by means of the second-order Adam-Bashforth scheme. This allows us to encapsulate the control algorithm within an ad hoc created C++ class of boundary conditions derived from the general OpenFOAM class named fixedValue, making the control implementation independent from the specific choice of the OpenFOAM flow solver and thus ready available for a wide range of flow simulations. Once computed by means of the finite-element discretization, the feedback gain field $\mathbf{u}_{k}(x, y)$ is interpolated on the hybrid finite-volume mesh and given in input to the control algorithm in the U-RANS simulation. The whole numerical setup has been first validated on the cylinder wake at low Reynolds numbers, where the MCE approach has been shown to completely suppress the vortex-shedding. ${ }^{30}$ For such test case, a coarse rectangular unstructured mesh composed of 38646 triangles is employed, with a minimum mesh size of $\approx 0.02$. The cylinder is placed at a distance of 15 diameters from the inlet and the lateral boundaries and of 30 diameters from the outflow boundary. A uniform streamwise velocity is assigned at the inlet, while on the remaining portions of the external boundary $\boldsymbol{\nabla} \boldsymbol{U} \cdot \hat{\boldsymbol{n}}=\mathbf{0}$ is assumed. For $R e=50$, the unstable eigenvalue extracted from the global stability analysis of the inherent base flow is (a)

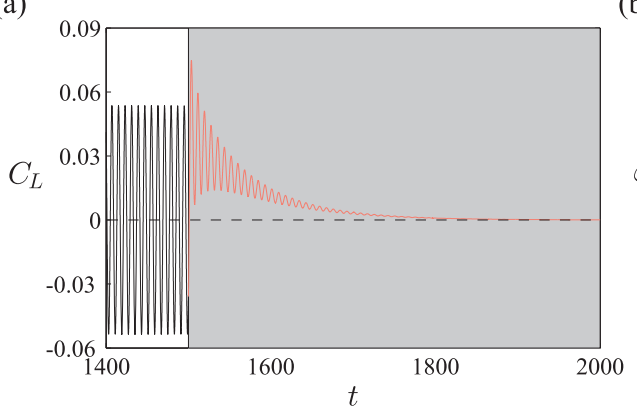

(b)

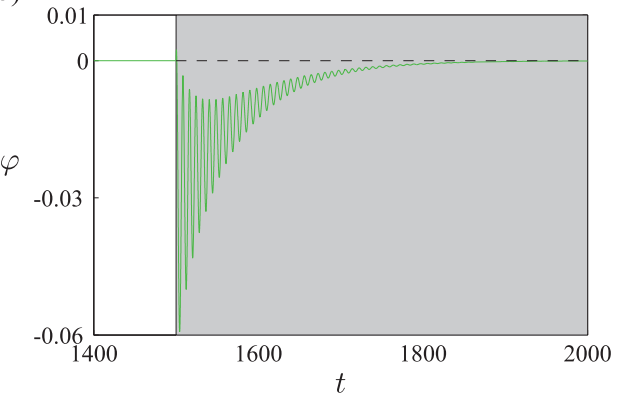

FIG. 15. MCE control of the laminar cylinder wake at $R e=50$. (a) Lift coefficient $C_{L}(t)$. (b) Control variable $\varphi(t)$ corresponding to the cylinder angular velocity which is assumed positive in the courter-clockwise direction. The control starts at the nondimensional time $t=1500$. 
(a)

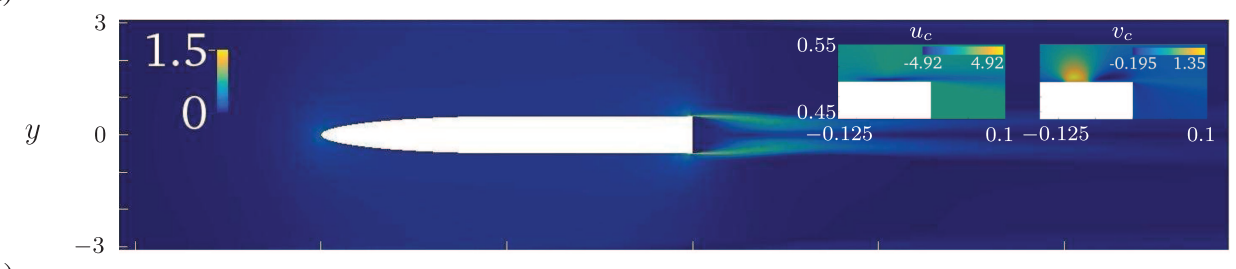

(b)

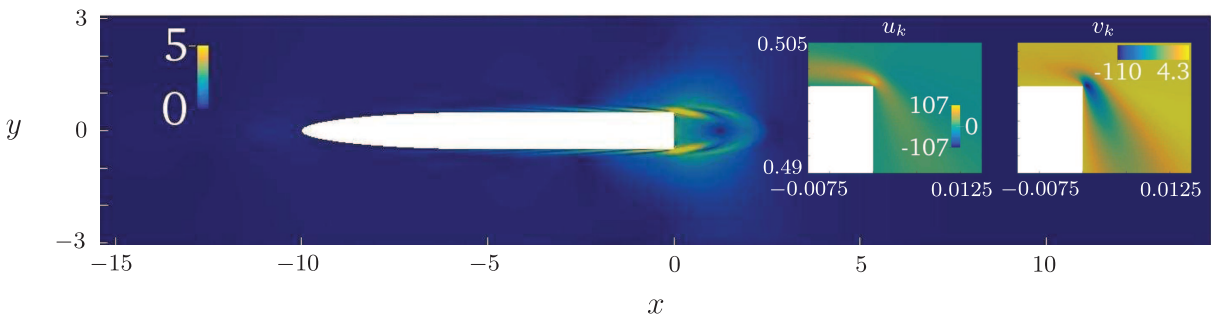

FIG. 16. Active feedback control of the confined turbulent flow past the thickflat plate. (a) Magnitude of the lifting velocity field, $\left\|\boldsymbol{u}_{c}(x, y)\right\|$. (b) Magnitude of the feedback gain field, $\left\|\boldsymbol{u}_{k}(x, y)\right\|$. Note that in both panels the colormap has been saturated for visualization purpose, highlighting the near-wake flow structure. The insets in each panel illustrate a detailed view of the $x$ and $y$ components of the corresponding vector field in the neighbourhood of the thick-flat plate blunt edge, using the corresponding full colormap. $\lambda=1.597 \times 10^{-2}+0.7454 i$ which compares well with the value reported by Carini, Pralits and Luchini. ${ }^{30}$ The same occurs for the corresponding direct and adjoint modes (not shown here). As done in the aforementioned study, the unsteady wake is controlled by means of angular oscillations of the cylinder surface around its axis, with $\varphi(t)$ representing the cylinder angular velocity, which is assumed positive in the courter-clockwise direction. The computed value of $\alpha, \tilde{\alpha}$ and $\beta$ are reported in Table VI. In particular $\beta \approx 0$ which follows from the fact that the control gain field $\boldsymbol{u}_{k}$ displays the opposite symmetry with respect to the base flow, i.e., it is antisymmetric with respect to the flow centreline. The computed MCE control is turned on during the fullydeveloped vortex-shedding regime and the time traces of $C_{L}(t)$ and $\varphi(t)$ illustrated in Fig. 15 confirm that the flow instability is completely suppressed and the base flow state restored.

\section{B. Results}

The solution of the lifting problem Eq. (20) is illustrated in Fig. 16(a) by means of the magnitude of the related velocity field. In addition, a detailed view of the velocity components, $u_{c}$ and $v_{c}$, in the neighbourhood of the control boundary $\Gamma_{c}$ is reported. A similar representation is also adopted for the feedback gain field $\boldsymbol{u}_{k}(x, y)$ in Fig. 16(b). Note that, for visualization purpose, the colormaps associated with $\left\|\boldsymbol{u}_{c}(x, y)\right\|$ and $\left\|\boldsymbol{u}_{k}(x, y)\right\|$ have been saturated to a value lower than the maximum one. Both vector fields are anti-symmetric with respect to the $x$-axis, consistently with the employed anti-phase blowing/suction actuation. Not surprisingly, $\boldsymbol{u}_{k}(x, y)$ displays a spatial structure which is very similar to the one of the unstable adjoint mode extracted from the stability analysis of the S-RANS mean flow (not shown here). Correspondingly, the highest gains are reached within a small region close to the

(a)

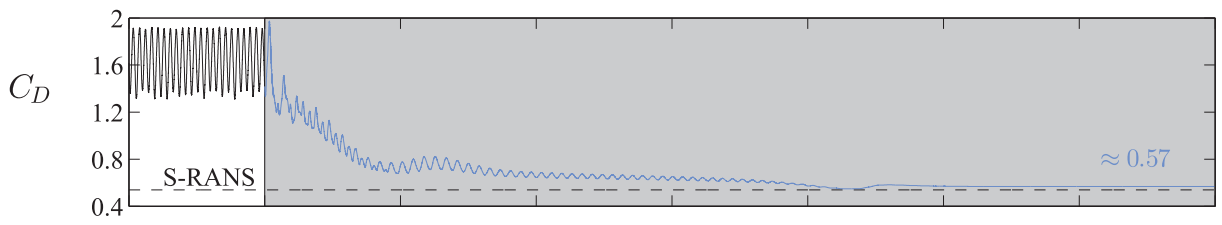

(b)

$C_{L}$

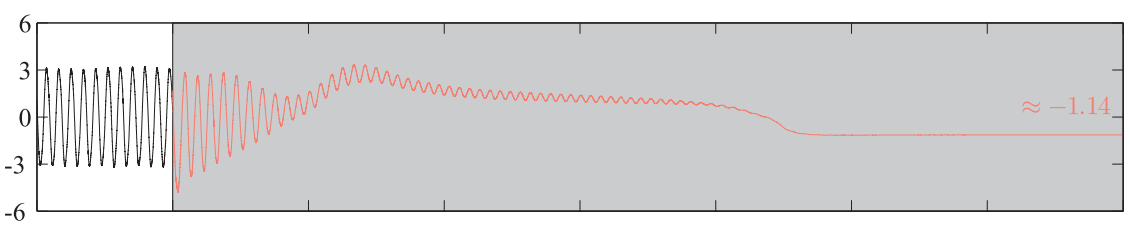

(c)
FIG. 17. Active feedback control of the confined turbulent flow past the thickflat plate: time traces of the thick-flat plate aerodynamic coefficients and of the control amplitude $\varphi(t)$. (a) Drag coefficient, $C_{D}$. (b) Lift coefficient, $C_{L}$. (c) Control amplitude $\varphi(t)$. 


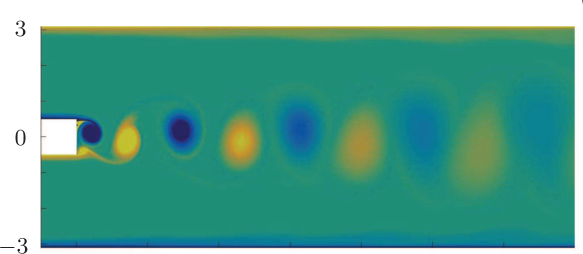

(d)
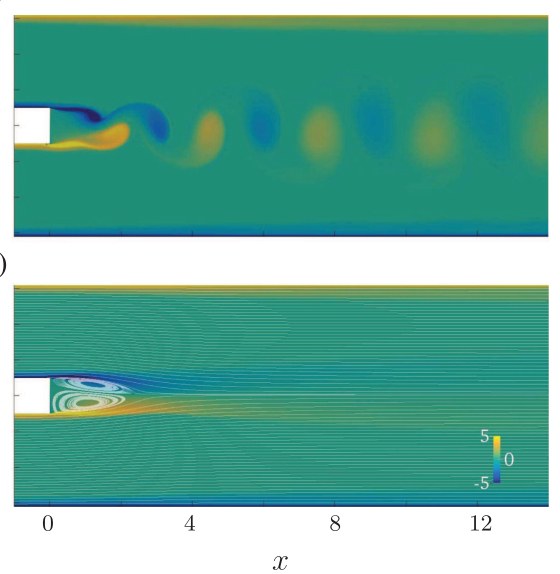

FIG. 18. Active feedback control of the confined turbulent flow past the thickflat plate: U-RANS vorticity snapshots at various time instants during the control. (a) $t=380$. (b) $t=440$. (c) $t=560$. (d) $t=680$. Note that the same saturated color-scale has been used for all the panels. In addition in panel (d), the flow streamlines are also illustrated, in order to better highlight the asymmetric structure of the final steady wake. thick flat-plate blunt edges, these values being almost two orders of magnitude greater than those attained in the nearwake, where, however, larger low-frequency velocity fluctuations are expected.

The feedback control law Eq. (27) is now introduced into the U-RANS simulations and applied directly to the fully developed vortex-shedding regime reached in the uncontrolled case, Fig. 7. The values of the control coefficients $\alpha, \tilde{\alpha}$ and $\beta$ are listed in Table VI. As expected from symmetry considerations, $\beta \approx 0$ while with respect to the circular cylinder test case the values of $\alpha$ and $\tilde{\alpha}$ are $\approx 1$ order of magnitude greater. The obtained control results are illustrated in Fig. 17, by means of the time traces of the aerodynamic force coefficients and of $\varphi(t)$, and in Fig. 18, by means of vorticity snapshots taken at different time instants. With reference to Fig. 17(a), after a first transient of $\approx 40$ time units from the control start-up, both the oscillation amplitude of $C_{D}(t)$ and its local mean value result greatly reduced and, finally, the $C_{D}(t)$ slowly converges toward a constant value, slightly above the one associated with the $\mathrm{S}$ RANS solution. Similarly, the oscillation amplitude of $C_{L}(t)$ finally reduces to zero, as shown in Fig. 17(b). However the asymptotic flow state is characterized by a constant negative lift force due to a constant blowing (suction) from the upper (lower) body surface at $\approx 17 \%$ of the free-stream velocity, as observed from the time trace of $\varphi(t)$ in Fig. 17(c). Note that the maximum blowing/suction velocity reached during the transient is of nearly 2 times the free-stream velocity. The control is able to completely suppress the flow unsteadiness associated with the vortex-shedding instability and the corresponding flow evolution is illustrated in Fig. 18. The vortex-rollup is first delayed downstream of the body stern, resulting in the formation of a "dead water" region, Fig. 18(b), which produces an increase of the base pressure and thus considerably reduces the body drag after the first transient of $\approx 40$. It is interesting to note that this physical mechanism is very similar to the one described by Pastoor et $a l .{ }^{45}$ in their active control experiment of the turbulent flow past a D-shaped cylinder. Then the alternating character of the wake and the associated vortex structures result progressively mitigated, Fig. 18(c), until a slightly asymmetric steady wake is established under the action of a constant non-zero blowing-suction actuation, showing nearly the same recirculation length of the target
S-RANS solution. The asymmetry of the asymptotic flow state of the fully nonlinear closed-loop system can be associated to the integral character itself of the feedback control law, Eq. (28), as well as to a destabilization of one of the modes lying on the real axis of the linearized (quasi-laminar mixed) flow spectrum, Fig. 9(b), through a "water-bed" effect.

\section{SUMMARY AND CONCLUSIONS}

We presented in this study a linear stability analysis of the confined turbulent mean flow past an elongated D-shaped cylinder using a quasi-laminar approach and its mixed variant. ${ }^{27}$ In analogy with previous works, ${ }^{25,27,28}$ we found that the vortex-shedding frequency measured in the experiments is very well captured by the leading unstable global mode, especially when the eddy viscosity is introduced in the stability equations. Despite the discrepancies observed between the measured and the computed mean wake, a good qualitative agreement is found when comparing the corresponding sensitivity maps in the near-wake region, where PIV measurements of the flow are performed. Both for the direct-adjoint product, i.e., the wavemaker, and the sensitivity to generic mean-flow variations, regions of high intensity display as thin layers along the boundary of the recirculation bubble. Similar spatial structures were described by Camarri, Fallenius and Fransson ${ }^{28}$ in their stability investigation of the turbulent mean wake behind a circular cylinder with transpiration. However, the visual inspection of these fields when extended to the whole spatial domain reveals that huge values of receptivity are present at the trailing edge of the body within a tiny region of size of $\mathcal{O}\left(H^{-3}\right)$. The core of the instability is thus composed by two distinct regions, the one located at the downstream edge of the recirculation bubble, common to the circular cylinder wake, and a second one located at the blunt edge of the thick flat-plate. These findings, which have not been pointed out in the aforementioned studies over a D-shaped geometry, suggest that particular care should be taken in the global stability analysis of PIV measured flow field when fixed separation points are left outside of the measurement window. In addition, the comparison between the quasi-laminar and the quasi-laminar mixed results show that the turbulent viscosity has a greater impact on the near-wake sensitivity structures rather than on 
the trailing-edge ones, with the former resulting appreciably smoothed.

As an additional application of the obtained stability results we explored the possibility to control the low-frequency oscillations of the wake. With respect to the works of Meliga Pujals and Serre ${ }^{25}$ and of Mettot, Sipp and Bézard, ${ }^{27}$ we considered here the design of an active feedback control using the linear optimal control theory. An original extension of the MCE technique ${ }^{29,30}$ to turbulent flow oscillators has been proposed under the frame of their RANS modelling. When applied directly to the fully developed vortex-shedding in the U-RANS simulations, the derived full-information control achieves a complete suppression of the wake oscillations. The control action results in a synchronization between the lower and upper shear layers with a mechanism similar to the one experimentally investigated by Pastoor et al. ${ }^{45}$ Such results indirectly support the relevance of the mean-flow stability and sensitivity analyses. Nevertheless attention need to be paid to the proper interpretation of the control performance. Although from a physical viewpoint a complete suppression of the vortex-shedding at high Reynolds numbers is rather unrealistic, the control results suggest that the computed gain field can select the most relevant "phase-averaged" velocity information for the effective mitigation of the global instability. Therefore the spatial distribution of the feedback gains can be used to guide the design of a real control experiment where phase-averaged measurements of the flow are available. In particular, for the present geometry, the structure of the gain field, as inherited from the underlying adjoint mode, can be used, at least in principle, to design a simple proportional control exploiting localized flow measurements at the body stern, where the highest gain values are found. These issues will be considered in a future work more focused on flow control aspects.

\section{ACKNOWLEDGMENTS}

The authors wish to acknowledge the financial support of the RTRA-STAE Foundation of Toulouse under the "CARPE" project and the Calmip computing centre of Toulouse for the availability of high-performance computing resources under the "P0824" project. This work was developed when Dr. J. O. Pralits served as invited Professor of Fluid Mechanics at the Paul Sabatier University of Toulouse (UPS). The support of UPS is also gratefully acknowledged. Finally, the authors are also grateful to Dr. Joel Guerrero from the Dipartimento di Ingegneria Civile, Chimica e Ambientale of the Università degli Studi di Genova for his helpful advices on the numerical simulations using OpenFOAM.

${ }^{1}$ M. Gad-el Hak, Flow Control: Passive, Active and Reactive Flow Management (Cambridge University Press, 2000).

${ }^{2}$ H. Choi, W. Jeon, and J. Kim, "Control of flow over bluff body," Annu. Rev. Fluid Mech. 40, 113-139 (2008).

${ }^{3}$ J. Wu, Y. L. Qiu, C. Shu, and N. Zhao, "Flow control of a circular cylinder by using an attached flexible filament," Phys. Fluids 26, 103601 (2014).

${ }^{4}$ J. Kim and T. R. Bewley, "A linear system approach to flow control," Annu. Rev. Fluid Mech. 39, 383-417 (2007)

${ }^{5}$ D. Sipp and P. J. Schmid, "Linear closed-loop control of fluid instabilities and noise-induced perturbations: A review of approaches and tools," Appl. Mech. Rev. 68, 020801 (2016).
${ }^{6}$ A. Barbagallo, D. Sipp, and P. J. Schmid, "Closed-loop control of an open cavity flow using reduced-order models," J. Fluid Mech. 641, 1-50 (2009). ${ }^{7}$ S. Ahuja and C. W. Rowley, "Feedback control of unstable steady state of flow past a flat plate using reduced-order estimators," J. Fluid Mech. 645, 447-478 (2010).

${ }^{8}$ A. Barbagallo, G. Dergham, D. Sipp, P. J. Schmid, and J.-C. Robinet, "Closed-loop control of unsteadiness over a rounded backward-facing step," J. Fluid Mech. 703, 326-362 (2012).

${ }^{9}$ O. Semeraro, J. O. Pralits, C. W. Rowley, and D. S. Henningson, "Riccatiless approach for optimal control and estimation: An application to twodimensional boundary layers," J. Fluid Mech. 731, 394-417 (2013).

${ }^{10}$ F. Giannetti and P. Luchini, "Structural sensitivity of the first instability of the cylinder wake," J. Fluid Mech. 581, 167-197 (2007).

${ }^{11}$ J. O. Pralits, L. Brandt, and F. Giannetti, "Instability and sensitivity of the flow around a rotating circular cylinder," J. Fluid Mech. 650, 1-24 (2010).

${ }^{12}$ I. Lashgari, J. O. Pralits, F. Giannetti, and L. Brandt, "First instability of the flow of shear-thinning and shear-thickening fluids past a circular cylinder," J. Fluid Mech. 701, 201-227 (2012).

${ }^{13}$ M. Carini, F. Giannetti, and F. Auteri, "First instability and structural sensitivity of the flow past two side-by-side cylinders," J. Fluid Mech. 749, 627-648 (2014).

${ }^{14}$ V. Citro, F. Giannetti, and J. O. Pralits, "Three-dimensional stability, receptivity and sensitivity of non-Newtonian flows inside open cavities," Fluid Dyn. Res. 47, 015503 (2015).

${ }^{15}$ M. Carini, F. Giannetti, and F. Auteri, "On the origin of the flip-flop instability of two side-by-side cylinder wakes," J. Fluid Mech. 742, 552-576 (2014).

${ }^{16}$ P. J. Strykowski and K. R. Sreenivasan, "On the formation and suppression of vortex 'shedding' at low Reynolds numbers,' J. Fluid Mech. 218, 71-107 (1990).

${ }^{17}$ O. Marquet, D. Sipp, and L. Jacquin, "Sensitivity analysis and passive control of cylinder flow," J. Fluid Mech. 615, 221-252 (2008).

${ }^{18}$ D. Barkley, "Linear analysis of the cylinder wake mean flow," Europhys. Lett. 75, 750-756 (2006)

${ }^{19}$ J. D. Crouch, A. Garbaruk, and D. Magidov, "Predicting the onset of flow unsteadiness based on global instability," J. Comput. Phys. 224, 924-940 (2007).

${ }^{20} \mathrm{~K}$. Gudmundsson and T. Colonius, "Instability wave models for the nearfield fluctuations of turbulent jets," J. Fluid Mech. 689, 97-128 (2011).

${ }^{21}$ W. V. R. Malkus, "Outline of a theory of turbulent shear flow," J. Fluid Mech. 1, 521-539 (1956).

${ }^{22}$ V. Mantič-Lugo, C. Arratia, and F. Gallaire, "Self-consistent mean flow description of the nonlinear saturation of the vortex shedding in the cylinder wake," Phys. Rev. Lett 113, 084501 (2014)

${ }^{23}$ S. E. Turton, L. S. Tuckerman, and D. Barkley, "Prediction of frequencies in thermosolutal convection from mean flows," Phys. Rev. E 91, 043009 (2015).

${ }^{24}$ S. Beneddine, D. Sipp, A. Arnault, J. Dandois, and L. Lesshafft, "Conditions for validity of mean flow stability analysis,” J. Fluid Mech. 798, 485-504 (2016).

${ }^{25}$ P. Meliga, G. Pujals, and E. Serre, "Sensitivity of 2-D turbulent flow past a D-shaped cylinder using global stability," Phys. Fluids 24, 061701 (2012).

${ }^{26}$ V. Parezanović and O. Cadot, "Experimental sensitivity analysis of the global properties of a two-dimensional turbulent wake," J. Fluid Mech. 693, 115-149 (2012).

${ }^{27}$ C. Mettot, D. Sipp, and H. Bézard, "Quasi-laminar stability and sensitivity analyses for turbulent flows: Prediction of low-frequency unsteadiness and passive control," Phys. Fluids 26, 045112 (2014).

${ }^{28}$ S. Camarri, B. E. G. Fallenius, and J. H. M. Fransson, "Stability analysis of experimental flow fields behind a porous cylinder for the investigation of the large-scale wake vortices," J. Fluid Mech. 715, 499-536 (2013).

${ }^{29}$ T. R. Bewley, J. O. Pralits, and P. Luchini, "Minimal-energy control feedback for stabilization of bluff-body wakes," in Proceeding FEDSM200231048, BBVIV5, Fifth Conference on Bluff Body Wakes and Vortex-Induced Vibrations (Bahia, Brazil, 2007).

${ }^{30}$ M. Carini, J. O. Pralits, and P. Luchini, "Feedback control of vortex shedding using a full-order optimal compensator," J. Fluids Struct. 53, 15-25 (2015).

${ }^{31}$ Source code and Documentation, available at http://www.openfoam.org, 2016.

${ }^{32}$ F. R. Menter, "Two-equation eddy-viscosity turbulence models for engineering applications," AIAA J. 32, 1598-1605 (1994).

${ }^{33}$ S. V. Patankar and D. B. Spalding, "A calculation procedure for heat, mass and momentum transfer in three-dimensional parabolic flows," Int. J. Heat Mass Transfer 15, 1787-1806 (1972). 
${ }^{34}$ R. I. Issa, "Solution of the implicitly discretised fluid flow equations by operator-splitting," J. Comput. Phys. 62, 40-65 (1986).

${ }^{35} \mathrm{~F}$. Menter, M. Kuntz, and R. Langtry, "Ten years of industrial experience with the SST turbulence model," in Turbulence, Heat and Mass Transfer 4, edited by K. Hanjalic, Y. Nagano, and M. Tummers (Begell House, 2003), pp. 625-632.

${ }^{36} \mathrm{E}$. Robertson, V. Choudhury, S. Bhushan, and D. Walters, "Validation of openfoam numerical methods and turbulence models for incompressible bluff body flows," Comput. Fluids 123, 122-145 (2015).

${ }^{37}$ D. A. Lyn, S. Einav, W. Rodi, and J. H. Park, "A laser-doppler velocimetry study of ensemble-averaged characteristics of the turbulent near wake of a square cylinder," J. Fluid Mech. 304, 285-319 (1995).

${ }^{38}$ B. E. Lee, "The effect of turbulence on the surface pressure field of a square prism," J. Fluid Mech. 69, 263-282 (1975).

${ }^{39}$ W. Rodi, J. H. Ferziger, M. Breuer, and M. Pourquie, "Status of largeeddy simulation: Results of a workshop," J. Fluids Eng. 119, 248 (1997).

${ }^{40}$ M. Minguez, C. Brun, R. Pasquetti, and E. Serre, "Experimental and highorder LES analysis of the flow in near-wall region of a square cylinder," Int. J. Heat Fluid Flow 32, 558 (2011).

${ }^{41}$ G. Iaccarino, A. Ooi, P. A. Durbin, and M. Behnia, "Reynolds averaged simulation of unsteady separated flow," Int. J. Heat Fluid Flow 24, 147 (2003).

${ }^{42} \mathrm{G}$. Bosch and W. Rodi, "Simulation of vortex shedding past a square cylinder with different turbulence models," Int. J. Numer. Methods Fluids 28, 601616 (1998)

${ }^{43}$ P. Meliga, E. Boujo, G. Pujals, and F. Gallaire, "Sensitivity of aerodynamic forces in laminar and turbulent flow past a square cylinder," Phys. Fluids 26, 104101 (2014).

${ }^{44}$ J. B. Barlow, W. H. Rae, and A. Pope, Low-Speed Wind Tunnel Testing, 3rd ed. (Wiley, 1999).

${ }^{45}$ M. Pastoor, L. Henning, B. R. Noak, R. King, and G. Tadmor, "Feedback shear layer control for bluff body drag reduction," J. Fluid Mech. 608, 161196 (2008).

${ }^{46}$ A. Naghib-Lahouti, P. Lavoie, and H. Hangan, "Wake instabilities of a blunt trailing edge profiled body at intermediate Reynolds numbers," Exp. Fluids 55, 1-15 (2014).
${ }^{47}$ M. Bull, Y. Li, and J. Pickles, "Effects of boundary layer transition on vortex shedding from thick plates with faired leading edge and square trailing edge," in Proceedings of the 12th Australasian fluid mechanics conferenceSydney, Australia, 2007.

${ }^{48}$ M. S. Alnæs, J. Blechta, J. Hake, A. Johansson, B. Kehlet, A. Logg, C. Richardson, J. Ring, M. E. Rognes, and G. N. Wells, "The FEniCS project version 1.5," Arch. Numer. Software 3, 9-23 (2015).

${ }^{49}$ R. Amestoy, I. Duff, and J.-Y. L'Excellent, "Multifrontal parallel distributed symmetric and unsymmetric solvers," Comput. Methods Appl. Mech. Eng. 184, 501-520 (2000)

${ }^{50}$ P. R. Amestoy, I. S. Duff, J. Koster, and J. Y. L'Excellent, "A fully asynchronous multifrontal solver using distributed dynamic scheduling," SIAM J. Matrix Anal. Appl. 23, 15-41 (2001).

${ }^{51}$ V. Hernandez, J. E. Roman, and V. Vidal, "SLEPc: A scalable and flexible toolkit for the solution of eigenvalue problems," ACM Trans. Math. Software 31, 351-362 (2005).

${ }^{52}$ J. E. Roman, C. Campos, E. Romero, and A. Tomas, "SLEPc users manual," Technical Report No. DSIC-II/24/02-Revision 3.7 (D. Sistemes Informàtics i Computació, Universitat Politècnica de València, 2016).

${ }^{53}$ D. Sipp and A. Lebedev, "Global stability of base and mean flows: A general approach and its applications to cylinder and open cavity flows," J. Fluids Mech. 593, 333-358 (2007).

${ }^{54} \mathrm{C}$. Airiau, "Noise sensitivity in a 2D compressible boundary layer flow past a cavity," 20ème Congrès Français de Mécanique, 28 août/2 September 2011-25044, Besançon, France, 2011.

${ }^{55}$ C. Mettot, F. Renac, and D. Sipp, "Computation of eigenvalue sensitivity to base flow modifications in a discrete framework: Application to open-loop control," J. Comput. Phys. 269, 234-258 (2014).

${ }^{56} \mathrm{E}$. Boujo and F. Gallaire, "Sensitivity and open-loop control of stochastic response in a noise amplifier flow: The backwardfacing step," J. Fluid Mech. 762, 361-392 (2015)

${ }^{57}$ J. B. Burl, Linear Optimal Control: $\mathcal{H}_{2}$ and $\mathcal{H}_{\infty}$ Methods (Addison-Wesley Longman Publishing, Co., Boston, Massachusetts, USA, 1998).

${ }^{58}$ In the sense of U-RANS simulations.

${ }^{59}$ J. O. Pralits, T. R. Bewley, and P. Luchini, "Feedback stabilization of the wake behind a steady cylinder," in 7th ERCOFTAC SIG 33-FLUBIO Workshop on Open Issues in Transition and Flow Control, Genova, Italy, 2008 